\title{
Review Article \\ Crosstalk between Calcium and ROS in Pathophysiological Conditions
}

\author{
Simona Feno, Gaia Butera, Denis Vecellio Reane, Rosario Rizzuto $(\mathbb{D}$, and Anna Raffaello \\ Department of Biomedical Sciences, University of Padova, via U. Bassi 58/b, 35131 Padova, Italy \\ Correspondence should be addressed to Rosario Rizzuto; rosario.rizzuto@unipd.it and Anna Raffaello; anna.raffaello@unipd.it
}

Received 24 January 2019; Accepted 1 April 2019; Published 24 April 2019

Guest Editor: Ulrike Hendgen-Cotta

Copyright (c) 2019 Simona Feno et al. This is an open access article distributed under the Creative Commons Attribution License, which permits unrestricted use, distribution, and reproduction in any medium, provided the original work is properly cited.

Calcium ions are highly versatile intracellular signals that regulate many cellular processes. The key to achieving this pleiotropic role is the spatiotemporal control of calcium concentration evoked by an extensive molecular repertoire of signalling components. Among these, reactive oxygen species (ROS) signalling, together with calcium signalling, plays a crucial role in controlling several physiopathological events. Although initially considered detrimental by-products of aerobic metabolism, it is now widely accepted that ROS, in subtoxic levels, act as signalling molecules. However, dysfunctions in the mechanisms controlling the physiological ROS concentration affect cellular homeostasis, leading to the pathogenesis of various disorders.

\section{Calcium Homeostasis}

Calcium ions $\left(\mathrm{Ca}^{2+}\right)$ are one of the most crucial intracellular second messengers, involved in a plethora of cellular functions including cell survival and death, muscle contraction, regulation of metabolism, and gene expression [1]. To control these highly specialized functions, cells have developed sophisticated mechanisms to decode frequency-encoded $\mathrm{Ca}^{2+}$ signals [1].

The spatiotemporal regulation of cytosolic $\mathrm{Ca}^{2+}$ concentration $\left(\left[\mathrm{Ca}^{2+}\right]_{\mathrm{cyt}}\right)$ relies on two key requirements. The first is the cooperation of two different sources of $\mathrm{Ca}^{2+}$ in the generation of $\left[\mathrm{Ca}^{2+}\right]_{\mathrm{cyt}}$ fluctuations: the extracellular medium, a virtually unlimited reservoir with a $\left[\mathrm{Ca}^{2+}\right]$ of $\sim 1 \mathrm{mM}$ [2], and the intracellular stores which are endowed with a $\left[\mathrm{Ca}^{2+}\right]>100 \mu \mathrm{M}$, which allow rapid release of $\mathrm{Ca}^{2+}$ through store-resident channels [2]. The second requirement is the existence of a broad range of molecules that generate and decode $\left[\mathrm{Ca}^{2+}\right]_{\text {cyt }}$ variations, such as pumps, channels, $\mathrm{Ca}^{2+}$-binding signalling molecules, enzymes, and buffering proteins [2].

Once having entered the cytosol, $\mathrm{Ca}^{2+}$ exerts its allosteric regulatory effects on many enzymes and proteins, impacting nearly every aspect of cellular life [3]. This is corroborated by the amount of energy that cells invest to maintain this strictly regulated $\left[\mathrm{Ca}^{2+}\right]$. Importantly, while complex molecules can be chemically altered, the only mechanism that exerts control over $\mathrm{Ca}^{2+}$ are chelation, subcellular compartmentalization and cell extrusion. The consequence is a very steep $\left[\mathrm{Ca}^{2+}\right]$ gradient across the plasma membrane and the intracellular stores [3]. In resting cells, $\left[\mathrm{Ca}^{2+}\right]_{\mathrm{cyt}}$ are maintained within very low values of $\sim 100 \mathrm{nM}$, while the extracellular space generally presents a $\left[\mathrm{Ca}^{2+}\right]$ of over $1 \mathrm{mM}$ [2]. Different channels in the plasma membrane regulate $\mathrm{Ca}^{2+}$ entry from the extracellular space. Among these are the voltage-operated calcium channels (VOCCs), the receptoroperated calcium channels (ROCCs), the store-operated calcium channels (SOCCs), and the second messengeroperated calcium channels (SMOCs) that, according to the stimuli evoking channel activation, allow $\mathrm{Ca}^{2+}$ entry through the plasma membrane [3].

As mentioned above, $\mathrm{Ca}^{2+}$ is also efficiently stored in intracellular compartments that serve as the main sources of releasable $\mathrm{Ca}^{2+}$ for eliciting crucial cellular functions [3]. The most important intracellular store is the endoplasmic reticulum (ER) and its specialized counterpart in muscle 
cells, the sarcoplasmic reticulum (SR). In these compartments, $\left[\mathrm{Ca}^{2+}\right]$ can reach $\sim 0.8 \mathrm{mM}$, depending on the cell type. Rapid release of $\mathrm{Ca}^{2+}$ from these compartments ensures $\left[\mathrm{Ca}^{2+}\right]_{\text {cyt }}$ rises required for specific cellular functions [3] and is controlled by two large families of channels: the inositol 1,4,5-trisphosphate receptor (InsP3R) and ryanodine receptor (RYR) families [4].

The agonist of IP3R is generated by the phospholipase C (PLC) enzymatic activity. This enzyme usually undergoes a receptor-promoted activation, and it hydrolyses its substrate phosphatidylinositol 4,5-bisphosphate (PIP2) in diacylglycerol (DAG) and inositol 1,4,5-trisphosphate (InsP3) [5]. The interaction of InsP3 with its receptors (InsP3Rs) induces $\mathrm{Ca}^{2+}$ release to the cytosol [6]. $\mathrm{Ca}^{2+}$ itself regulates the InsP3Rs' open probability, activating InsP3Rs at increasing $\left[\mathrm{Ca}^{2+}\right]$ up to a specific $\left[\mathrm{Ca}^{2+}\right]$ threshold, above which further increases in $\left[\mathrm{Ca}^{2+}\right]$ play an inhibitory function [2]. The InsP3R family displays a broad tissue distribution and comprises three isoforms, InsP3R1, InsP3R2, and InsP3R3, which show different expression profiles among different tissues. Of note, InsP3R1 is most abundant in the central nervous system (CNS) and InsP3R2 is ubiquitously expressed among tissues and is the most abundant isoform in cardiac muscle [7]. InsP3Rs form heterotetramers, whose activity displays unique properties and responsiveness to ATP, $\mathrm{Ca}^{2+}$, and InsP3 [8].

RyRs are structurally and functionally analogous to InsP3Rs, although they have approximately twice the conductance and molecular mass of InsP3Rs. RyRs are transmembrane proteins located in the ER/SR membrane, activated by the alkaloid ryanodine and by $\mathrm{Ca}^{2+}$ itself. Although $\mathrm{Ca}^{2+}$ is a major triggering ligand, several other players modulate RyRs' activity, such as the dihydropyridine receptor (DHPR; also known as L-type $\mathrm{Ca}^{2+}$ channel, $\left.\mathrm{Ca}_{\mathrm{V}} 1.1 / 1.2\right)$, protein kinase A (PKA), calmodulin (CaM), $\mathrm{Ca}^{2+} /$ calmodulin-dependent protein kinase II (CaMKII), calsequestrin (CSQ), and the FK506-binding protein (FKBP12) [9]. Similarly to InsP3Rs, RyRs include three isoforms (RyR1-3), but, unlike the InsP3Rs that are widely expressed among tissues, RyR1-3 are almost exclusively expressed in excitable cell types. In detail, RyR1 is particularly enriched in skeletal muscle, RyR2 in cardiac muscle, and RyR3 is expressed more widely, although higher levels are found in the brain [6].

Once $\mathrm{Ca}^{2+}$ has carried out its signalling functions, it has to be rapidly removed from the cytosol by extrusion to the extracellular space or by compartmentalization to intracellular stores. This is achieved thanks to the activity of various pumps and exchangers, allowing intracellular $\left[\mathrm{Ca}^{2+}\right]$ to return to its resting condition [3]. ATPase pumps compartmentalize $\mathrm{Ca}^{2+}$ into the ER/SR stores via the activity of ER/SR $\mathrm{Ca}^{2+}$ ATPase pumps (SERCAs) or extrude $\mathrm{Ca}^{2+}$ in the extracellular milieu via plasma membrane $\mathrm{Ca}^{2+}$ ATPases (PMCA pumps) by exploiting ATP-derived energy. A second mechanism utilizes the electrochemical gradient of $\mathrm{Na}^{+}$across the plasma membrane to provide the energy to transport $\mathrm{Ca}^{2+}$ to the extracellular space through the $\mathrm{Na}^{+} / \mathrm{Ca}^{2+}$ (NCX) and $\mathrm{Na}^{+} / \mathrm{Ca}^{2+}-\mathrm{K}^{+}$exchangers (NCKX) [3].

In addition, many studies have highlighted a role in regulation of $\left[\mathrm{Ca}^{2+}\right]_{\mathrm{cyt}}$ also for other membrane-bound compartments such as the Golgi apparatus, endolysosomes, and mitochondria [6]. Among these organelles, mitochondria are recognized as crucial regulators of cellular $\mathrm{Ca}^{2+}$ homeostasis. Indeed, mitochondrial $\mathrm{Ca}^{2+}$ uptake regulates many cellular processes, controlling the delicate balance between cell survival and death [2]. Moreover, mitochondrial $\mathrm{Ca}^{2+}$ buffering is involved in the control of $\mathrm{Ca}^{2+}$ gradient in defined cellular domains [2]. This is possible thanks to a strategic localization of mitochondria to the $\mathrm{Ca}^{2+}$ release units of the ER/SR that contributes to shape both the amplitude and the spatiotemporal patterns of cellular $\mathrm{Ca}^{2+}$ responses [3].

1.1. Mitochondrial $\mathrm{Ca}^{2+}$ Signalling. Over the past 60 years, intense research has defined the basic properties of mitochondria in $\mathrm{Ca}^{2+}$ handling. These studies have highlighted the role of mitochondria in decoding the cytosolic $\mathrm{Ca}^{2+}$ oscillations and in the regulation of cellular $\mathrm{Ca}^{2+}$ homeostasis [3]. The first evidence that mitochondria can take up $\mathrm{Ca}^{2+}$ dates back to the 60s, when pioneering studies demonstrated that energized mitochondria can rapidly and efficiently accumulate $\mathrm{Ca}^{2+}[10,11]$. The formulation of the chemiosmotic theory, together with the measurement of the mitochondrial membrane potential $\left(\Delta \Psi_{\mathrm{m}}\right)$, led to the concept of an energetically favourable $\mathrm{Ca}^{2+}$ uptake mechanism $[12,13]$. The generation of an internal negative electrochemical gradient by the mitochondrial respiratory chain, indeed, provides the thermodynamic basis for cation accumulation into the organelle matrix [13]. However, further characterizations of the mitochondrial $\mathrm{Ca}^{2+}$ uptake demonstrated that, despite the high selectivity of the mitochondrial $\mathrm{Ca}^{2+}$ uniporter (MCU) for $\mathrm{Ca}^{2+}$, measured by direct mitoplast patch-clamp of mitoplasts (dissociation constant $(\mathrm{Kd}) \leq 2 \mathrm{nM}$ ) [14], the apparent mitochondrial affinity for $\mathrm{Ca}^{2+}$ was very low at physiological $\left[\mathrm{Ca}^{2+}\right][15]$. Since cytosolic $\left[\mathrm{Ca}^{2+}\right]$ is about $10-100 \mathrm{nM}$ in resting conditions and reaches values of $2-3 \mu \mathrm{M}$ during cell stimulation, the role of mitochondria in $\mathrm{Ca}^{2+}$ homeostasis was considered marginal. Therefore, the plasma membrane and the ER became the major players in the $\mathrm{Ca}^{2+}$ signalling scene [15]. The situation reversed when tools to perform reliable measurement of $\left[\mathrm{Ca}^{2+}\right]$ in intact living cells were developed, allowing to uncover the role of mitochondria in $\mathrm{Ca}^{2+}$ handling $[16,17]$. Indeed, while $\left[\mathrm{Ca}^{2+}\right]_{\text {mit }}$ in basal resting condition is very low, comparable to the cytosolic one (10-100 nM), upon cell stimulation, mitochondria are able to rapidly and efficiently accumulate $\mathrm{Ca}^{2+}$ at levels that exceed that of the bulk cytosol that, in some cell lines, can reach also $\left[\mathrm{Ca}^{2+}\right]$ of $100 \mu \mathrm{M}$ [16]. The discrepancy between the low affinity of mitochondrial $\mathrm{Ca}^{2+}$ uptake and the prompt response of mitochondria to $\left[\mathrm{Ca}^{2+}\right]$ increases was later solved by the demonstration that mitochondria are located in close proximity to the $\mathrm{Ca}^{2+}$ channels that elicit the rise in $\left[\mathrm{Ca}^{2+}\right]_{\mathrm{cyt}}$, the InsP3Rs, and the RYRs on the ER and SR $[18,19]$. Indeed, these quasi-synaptic junctions with the ER/SR membranes allow mitochondria to directly sense local high $\left[\mathrm{Ca}^{2+}\right]$ compatible with the low affinity of the MCU and that dissipates rapidly, thus preventing mitochondrial $\mathrm{Ca}^{2+}$ overload or vicious $\mathrm{Ca}^{2+}$ cycling across the mitochondrial membrane $[18,19]$. 
Although the process of mitochondrial calcium uptake is prevalently studied at the level of the solutes impermeable inner mitochondrial membrane (IMM), the ability of $\mathrm{Ca}^{2+}$ to cross the outer mitochondrial membrane (OMM) plays a crucial role. The OMM permeability to solutes is prevalently due to the high expression of the voltage-dependent ion channels (VDACs), permeable to solutes smaller than $5 \mathrm{kDa}$ and, thus, also $\mathrm{Ca}^{2+}[20]$. Three different VDAC isoforms, VDAC1, VDAC2, and VDAC3, have been identified. VDAC1, the best characterized isoform [21], acts as a mitochondrial gatekeeper, controlling the metabolic and energy crosstalk between the mitochondria and the rest of the cell [21]. Furthermore, it has been also shown that VDACs' expression levels can limit calcium accumulation inside the matrix. It was demonstrated, indeed, that VDAC overexpression augments agonist-dependent rises in $\left[\mathrm{Ca}^{2+}\right]_{\text {mit }}$, whereas VDAC downregulation has the opposite effect $[22,23]$.

1.2. The Mitochondrial $\mathrm{Ca}^{2+}$ Uniporter Complex: Structural and Functional Complexity. The molecular identity of the protein responsible for mitochondrial $\mathrm{Ca}^{2+}$ uptake, MCU, was uncovered only in 2011 by two different groups $[24,25]$, marking a turning point in the study of the pathophysiological roles of mitochondrial $\mathrm{Ca}^{2+}$ uptake. The characterization of the MCU revealed that this channel is a high-molecular-weight complex composed of both pore-forming and regulatory subunits [1].

From the primary amino acidic sequence analysis, MCU consists of two transmembrane domains spanning the IMM (Figure 1 and [1]). Soon after its discovery, it was clear that the MCU was part of a macromolecular complex since it lacks classical $\mathrm{Ca}^{2+}$-binding domains and the loop region that faces the intermembrane space (IMS) appears to be too small to contain regulatory elements [1]. This was confirmed by blue native gel separation experiments of purified mitochondria that display a high-molecular-weight complex containing MCU with an apparent molecular weight of about $450 \mathrm{kDa}$, suggesting that many other proteins are part of the channel [24, 26-28].

Recently, the MCU protein structure was solved by different laboratories. First, MCU was shown to be a pentamer of the MCU homolog from Caenorhabditis elegans deleted of the N-terminal domain, which was defined by using nuclear magnetic resonance (NMR) and negative-stain electron microscopy [29]. Recently, four independent groups characterized the structure full-length Fungi homologs of MCU by Cryo-EM and/or X-ray diffraction approaches [30-33]. Unlike the previous study, they found a tetrameric architecture. Since these Fungi MCU homologs share only about $40 \%$ of similarity with metazoan MCU, prevalently conserved in the transmembrane regions and in the coiled-coil domains, Baradaran and coworkers performed Cryo-EM studies also on zebrafish MCU homolog, which displays a higher similarity with human MCU (91\%). Although the resolution obtained is lower $(8.5 \AA)$, the overall structure is similar to that of Fungi MCU and also displays a tetrameric architecture [30]. Interestingly, the conserved DIME motif that connects the two transmembrane domains appears to be part of the second transmembrane domain and seems to confer $\mathrm{Ca}^{2+}$ selectivity to the MCU. The N-terminal domain is poorly conserved in these MCU homologs, but the human NTD of MCU was previously crystallized [34].

After the discovery of MCU, we have witnessed an explosion of studies aimed at clarifying the composition of the channel and the regulation of its activity. These studies demonstrated that three proteins compose the protein structure that spans the IMM: MCU, MCUb, and EMRE. Furthermore, three regulatory subunits were identified (MICU1, MICU2, and MICU3).

EMRE ("essential MCU regulator") is a $10 \mathrm{kDa}$, metazoan-specific protein with a single transmembrane domain that spans the IMM with a highly acidic carboxyl terminus (Figure 1 and [28]). This protein has been proposed to play a dual function in the regulation of MCU activity. First, it seems required for MCU channel activity since its silencing abrogates mitochondrial $\mathrm{Ca}^{2+}$ uptake [28], although experiments in the planar lipid bilayer demonstrated that mouse MCU alone is sufficient to give rise to $\mathrm{Ca}^{2+}$ currents [25]. Second, EMRE seems fundamental in mediating the interaction between MCU and the regulatory subunits MICU1 and MICU2 [28], although it has also been observed that MICU1 is sufficient to induce MCU channel activity [26]. In addition, in yeast cells that do not present mitochondrial $\mathrm{Ca}^{2+}$ uptake, the Dictyostelium discoideum MCU homolog conducts $\mathrm{Ca}^{2+}$ in the absence of an EMRE homolog while human MCU requires the presence of EMRE to act as a functional channel [35]. Very recently, it was shown that the acidic C-terminal domain functions as a matrix $\mathrm{Ca}^{2+}$ sensor that regulates the MCU activity. In this model, EMRE acts, together with MICU1, as a regulatory complex able to sense $\left[\mathrm{Ca}^{2+}\right]$ at both sides of IMM [36]. Nevertheless, these data were questioned by a study showing that EMRE displays a different topology across the IMM [37]. Future experiments will clarify the role of EMRE in the regulation of MCU channel activity.

MCUb is a MCU isoform conserved in most vertebrates and in many plants but absent in other organisms where the MCU is present (Figure 1 and [38]). MCU and MCUb share $50 \%$ sequence similarity, and each possesses two transmembrane domains separated by a short loop almost identical between the two [38]. Despite the huge sequence similarity in the transmembrane domains, MCUb displays altered ion permeation, given to two conserved amino acid substitutions in close proximity of the conserved DIME motif that drastically reduces the conductivity of the channel [38]. Specifically, the Arg 251 and Glu 256 residues are mutated in Trp and Val, respectively (R251W and E256V). These substitutions drastically reduce conductivity of the channel reducing $\left[\mathrm{Ca}^{2+}\right]_{\text {mit }}$ uptake [38]. According to this evidence, in living cells, the overexpression of MCUb reduces the amplitude of $\left[\mathrm{Ca}^{2+}\right]_{\text {mit }}$ transients evoked by agonist stimulation and MCUb silencing elicits the opposite effect, suggesting that this protein acts as a dominant-negative subunit that incorporates into the channel and reduces its activity [38]. Interestingly, MCU and MCUb expression profiles widely differ among tissues, possibly providing an intrinsic regulatory mechanism to set the mitochondrial responsiveness to $\mathrm{Ca}^{2+}$-mediated signals in a defined cell type [38]. Consistently, tissues characterized by low mitochondrial 


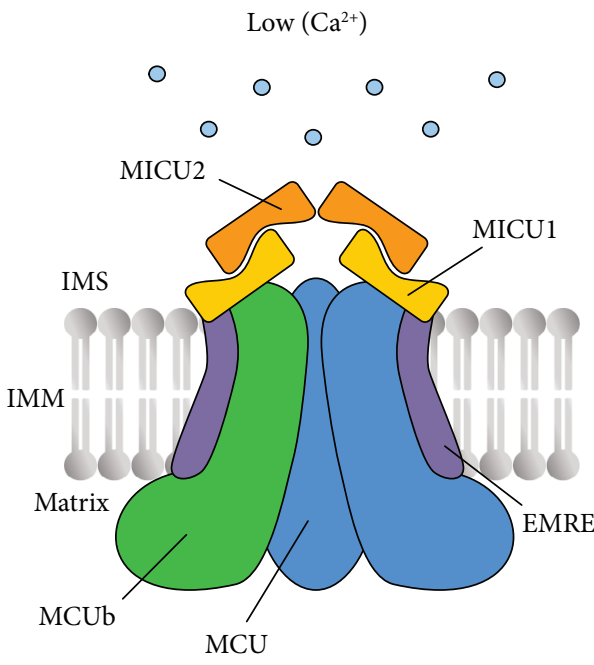

(a)

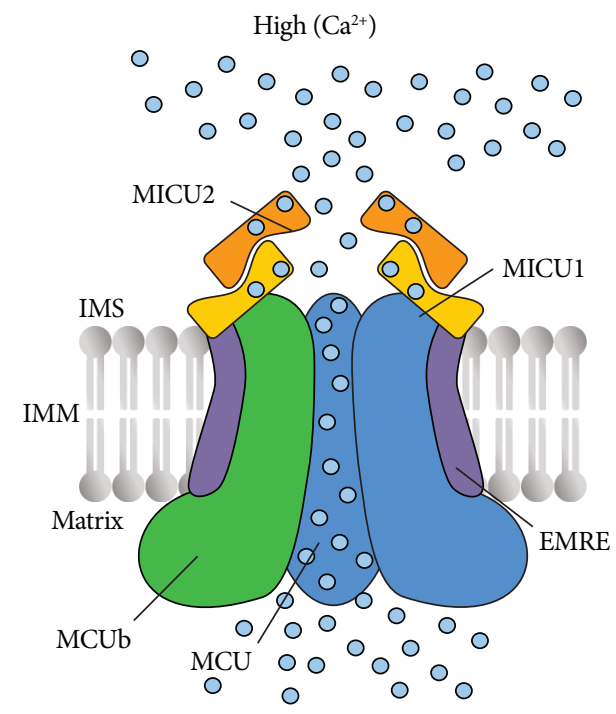

(b)

FIGURE 1: Schematic representation of the mitochondrial $\mathrm{Ca}^{2+}$ uniporter (MCU) complex. The MCU complex is composed by pore-forming subunits (that comprise the channel subunits MCU, the dominant-negative subunits MCUb, and the transmembrane regulator EMRE) and regulatory subunits (MICU1 and MICU2). MICU1 and MICU2 sense, through EF-hand domains, the increase of Ca ${ }^{2+}$ levels in the intermembrane space (IMS). In resting conditions (on the left), MICU1-MICU2 heterodimers act as gatekeeper of the channel, thus preventing $\mathrm{Ca}^{2+}$ vicious cycling and mitochondrial matrix overload. Increases in calcium concentration as a result of cell stimulation (on the right) not only release the inhibitory function of the MICU1-MICU2 heterodimers but also further stimulate MCU channel opening, ensuring the prompt response of mitochondrial metabolism to cell stimulation.

$\mathrm{Ca}^{2+}$ transients, such as the heart, exhibit a low MCU/MCUb ratio, while others, such as skeletal muscle, display a higher ratio and high mitochondrial $\mathrm{Ca}^{2+}$ uptake levels [38].

1.3. MCU-Associated Regulators. One of the key features of mitochondrial $\mathrm{Ca}^{2+}$ uptake is its sigmoidal response to extra-mitochondrial $\left[\mathrm{Ca}^{2+}\right]$. At resting $\left[\mathrm{Ca}^{2+}\right]_{\text {cyt }}$, mitochondrial $\mathrm{Ca}^{2+}$ uptake is inhibited, despite the steep $\Delta \Psi_{\mathrm{m}}$ [3]. This property prevents matrix $\mathrm{Ca}^{2+}$ overload and the dissipation of the $\Delta \Psi_{\mathrm{m}}$, leading to deleterious effects of $\mathrm{Ca}^{2+}$ cycling and matrix overload. At higher $\left[\mathrm{Ca}^{2+}\right]_{\text {cyt }}$, when cells are stimulated, mitochondria have to respond promptly, increasing the $\mathrm{Ca}^{2+}$-carrying capacity [3]. The lack of $\mathrm{Ca}^{2+}$-sensing domains in the MCU protein sequence, as mentioned above, suggested the existence of a highly sophisticated gatekeeping mechanism, including both negative modulators, acting at low $\left[\mathrm{Ca}^{2+}\right]$, and activators able to induce $\mathrm{Ca}^{2+}$ uptake during cell stimulation. Accordingly, it was shown that the regulation of the MCU complex activity is possible thanks to the MICU (mitochondrial calcium uptake) family of intermembrane space (IMS) proteins, composed by MICU1, MICU2, and MICU3 [39]. These three regulators share common features: they are localized to mitochondria, they display $\mathrm{EF}-$ hand $\mathrm{Ca}^{2+}$-binding domains in their protein sequence, and they interact with MCU (Figure 1) [39].

MICU1 (mitochondrial calcium uptake 1) was identified even before the identification of MCU as a critical modulator of mitochondrial $\mathrm{Ca}^{2+}$ uptake (Figure 1 and [40]). MICU1 was initially proposed to be required for mitochondrial $\mathrm{Ca}^{2+}$ uptake, since its silencing was sufficient to abolish mitochondrial $\mathrm{Ca}^{2+}$ entry in intact and permeabilized cells [40].
This evidence was questioned by other laboratories that showed that MICU1 silencing is sufficient to induce mitochondrial $\mathrm{Ca}^{2+}$ overload, suggesting that MICU1 could play a gatekeeping role in preventing mitochondrial $\mathrm{Ca}^{2+}$ uptake at low $\left[\mathrm{Ca}^{2+}\right]_{\mathrm{cyt}}$, while playing a minor role at higher $\left[\mathrm{Ca}^{2+}\right]_{\mathrm{cyt}}$ [41]. The identification of MICU1 loss-of-function mutations in patients affected by a disease characterized by proximal myopathy, learning difficulties, a progressive extrapyramidal movement disorder, and increased mitochondrial $\mathrm{Ca}^{2+}$ load supported this hypothesis [42]. The gatekeeper role of MICU1 was confirmed by Csordás and coworkers [43]. They also showed that silencing of MICU1 highly affects the cooperativity of mitochondrial $\mathrm{Ca}^{2+}$ uptake, thus hypothesizing that MICU1 could play a dual function depending on $\left[\mathrm{Ca}^{2+}\right]_{\mathrm{cyt}}$.

Very recently, Csordás et al.s' group dissected the mechanism that allows MICU1 to interact with MCU, to regulate mitochondrial $\mathrm{Ca}^{2+}$ entry and the sensitivity to ruthenium red/Ru360 (RuRed/Ru360), a compound that inhibits the activity of the uniporter [44]. Indeed, a structural and functional interaction of the DIME motif of MCU was reported, identified as the selectivity filter, with a domain of MICU1, named DID, as the DIMEinteracting domain. The interaction between these two domains appears to be fundamental for ensuring both the threshold and cooperative activation of the MCU complex-mediated $\mathrm{Ca}^{2+}$ uptake and thus to avoid mitochondrial $\mathrm{Ca}^{2+}$ overload [44]. Furthermore, the DID motif limits the access of RuRed/Ru360 to its target site in the DIME domain of MCU since MICU1 removal can sensitize mitochondria to inhibition by this compound, thus 
predicting a different RuRed/Ru360 sensitivity of the MCU complex in various tissues, in light of recent data on tissuespecific differences in MICU1 abundance relative to MCU [45].

In addition to MICU1, other MCU complex components have also been discovered. Two paralogs of MICU1, originating from a gene duplication event prior to vertebrate evolution, were identified: MICU2 (Figure 1 and [27]), which displays a tissue expression pattern similar to that of MICU1, and MICU3, whose expression is restricted to the nervous system (NS) and, at lower levels, to the skeletal muscle [27].

MICU2 discovery [27] helped to clarify the mechanism responsible for the sigmoidal response of the MCU to extramitochondrial $\left[\mathrm{Ca}^{2+}\right]$ that allows on the one hand minimal $\mathrm{Ca}^{2+}$ uptake in the presence of a very large driving force for cation accumulation thus preventing mitochondrial $\mathrm{Ca}^{2+}$ overload and on the other hand ensures rapid $\mathrm{Ca}^{2+}$ accumulation during cell stimulation. Importantly, MICU2 protein stability depends on that of MICU1 [26, 27, 46], since MICU1 silencing induces MICU2 protein degradation, suggesting that the effect of MICU1 silencing on mitochondrial $\mathrm{Ca}^{2+}$ uptake could be due also to the concomitant disappearance of MICU2 protein. Notably, MICU1 and MICU2 have been shown to form an obligate heterodimer through the formation of a disulphide bond [26], which is regulated by the mitochondrial oxidoreductase Mia40 [47].

MICU2 was demonstrated to act as the genuine gatekeeper of the MCU at low $\left[\mathrm{Ca}^{2+}\right]_{\mathrm{cyt}}[26]$. As soon as extramitochondrial $\left[\mathrm{Ca}^{2+}\right]$ increases, $\mathrm{Ca}^{2+}$-dependent MICU2 inhibition and MICU1 activation guarantee the prompt response of rapid mitochondrial $\mathrm{Ca}^{2+}$ accumulation (Figure 1 and [26]).

Recently, an alternative splice variant of MICU1, named MICU1.1, was identified and characterized [48]. It has been shown that the expression of this splice variant varies greatly among tissues. Indeed, MICU1.1 is present only in skeletal muscle, where it is the predominant isoform, and lower levels are found in the brain, suggesting tissue-specific functions. MICU1.1 is characterized by the addition of a micro-exon coding for four amino acids (EFWQ) far from the EF-hand domains, which greatly modifies the properties of the protein. In detail, MICU1.1 can bind $\mathrm{Ca}^{2+}$ one order of magnitude more efficiently than MICU1 and, when heterodimerized with MICU2, activates MCU current at lower $\left[\mathrm{Ca}^{2+}\right]$ than MICU1-MICU2 heterodimers [48].

How the MICU1.1 extra exon impact on MICU1 structure and modifies the $\mathrm{Ca}^{2+}$-binding affinity of the EF-hand domains remains unaccounted. In this regard, the domain that contains the extra exon was not resolved in the MICU1 crystal structure, suggesting that it is part of a highly flexible region [49]. This can suggest a putative role of this protein domain in protein-protein interactions, which can modify the MICU1 modulatory properties.

It was hypothesized that the inclusion of this splice variant in the MCU complex could represent an important mechanism in excitable tissues, where fast $\mathrm{Ca}^{2+}$ transients occur. Indeed, in skeletal muscle, the prevalent expression of MICU1.1 allows a prompter response of mitochondria metabolism to $\left[\mathrm{Ca}^{2+}\right][48]$, ensuring a sustained ATP production during contraction, since mitochondrial $\mathrm{Ca}^{2+}$ positively regulates the activity of three key dehydrogenases of the tricarboxylic acid (TCA) cycle: pyruvate, isocitrate, and $\alpha$-ketoglutarate dehydrogenases [50].

MICU3 shares a mitochondrial targeting sequence (MTS) at the amino terminus and two canonical $\mathrm{Ca}^{2+}$-binding EF-hand domains with MICU1 and MICU2 [27]. MICU3, unlike MICU1 and MICU2 that present a ubiquitous and strongly correlated expression pattern among tissues, is expressed only in the CNS and, at low levels, in skeletal muscle [27]. Recently, it was shown that MICU3 exists in a disulfide bond-mediated dimer only with MICU1 but not with itself or MICU2 and acts as a highly potent stimulator of MCU activity, with no gatekeeping function [51]. In this regard, it was shown that neurons simultaneously express both MICU1-MICU2 and MICU1-MICU3 heterodimers. The first avoids low vicious $\mathrm{Ca}^{2+}$ cycling in resting conditions; the latter anticipates MCU opening, activating organelle $\mathrm{Ca}^{2+}$ uptake even in the presence of small and rapid cytosolic $\mathrm{Ca}^{2+}$ signals. Thus, MICU3 in neurons allows enhancing MCU opening in order to guarantee organelle $\mathrm{Ca}^{2+}$ uptake also in response to small and fast increases of $\left[\mathrm{Ca}^{2+}\right]_{\text {cyt }}[51]$.

Finally, MCUR1, an IMM-integral protein, was initially reported to function as a regulator of the MCU complex [52], although this protein was not among the MCU interactors [28]. Furthermore, MCUR1 has a homolog in Saccharomyces cerevisiae, an organism that lacks mitochondrial $\mathrm{Ca}^{2+}$ uptake. Its role in MCU complex regulation is highly debated, since it has been shown, both in yeast and mammalian cells, that it is involved in complex IV assembly [53]. Furthermore, it has been reported that MCUR1 silencing causes a consistent drop of $\Delta \Psi_{\mathrm{m}}$, with consequent reduction of mitochondrial $\mathrm{Ca}^{2+}$ uptake [53].

Moreover, recently, Chaudhuri and coworkers found discordant results. Indeed, they found no significant changes in $\Delta \Psi_{\mathrm{m}}$ and no changes in mitochondrial $\mathrm{Ca}^{2+}$ uptake rates after manipulating MCUR1 expression, but they demonstrated that MCUR1 regulates the amount of $\mathrm{Ca}^{2+}$ required to induce the permeability transition [54].

Therefore, whether this protein controls directly the activity of MCU or whether it affects mitochondrial $\mathrm{Ca}^{2+}$ uptake by indirectly impinging on mitochondrial bioenergetics is still highly debated.

\section{2. $\mathrm{Ca}^{2+}$ and $\mathrm{ROS}$ as a Mutual Interplay}

The understanding of the role of mitochondria as integration points of different cellular signals, and the mechanisms through which mitochondria translate these stimuli in biological responses, represents a new challenge in biomedical research. As discussed above, the ability of mitochondria to accumulate $\mathrm{Ca}^{2+}$ is fundamental for tissue homeostasis [1]. However, mitochondrial $\mathrm{Ca}^{2+}$ overload leads to reduced ATP production and sustained opening of the MPTP, a high conductance channel, whose opening enables the release of proapoptotic mitochondrial components [55].

Matrix $\mathrm{Ca}^{2+}$, beyond a certain threshold, together with other causal factors, most notably oxidative stress, high 


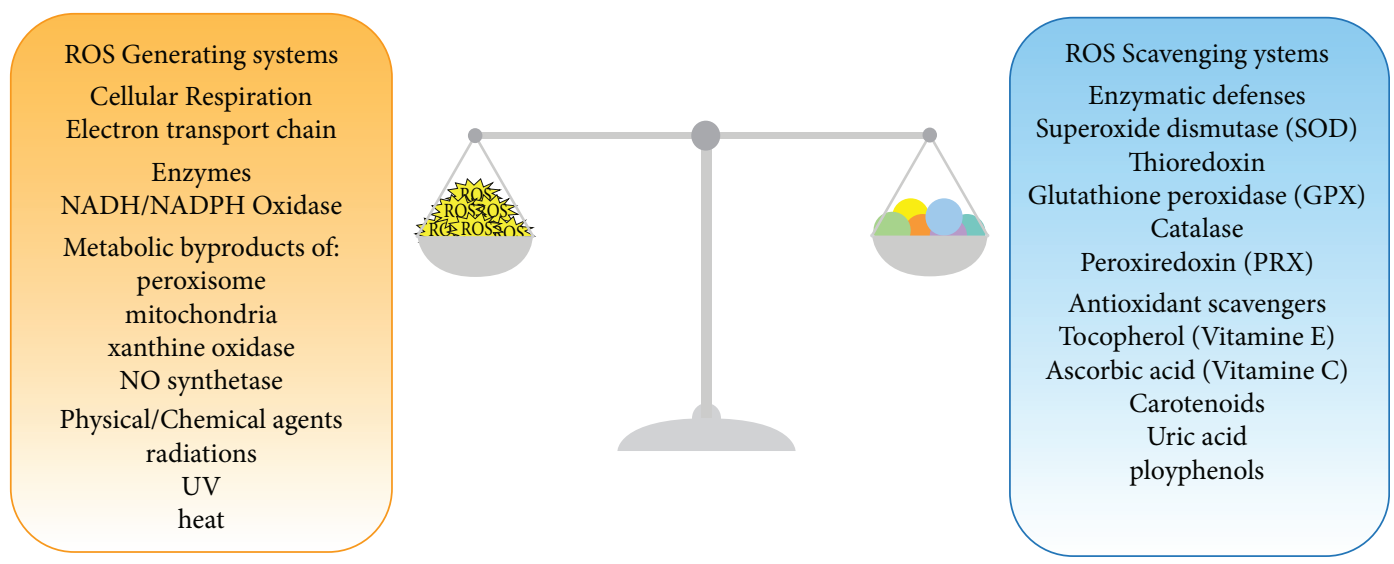

FIGURE 2: ROS production and scavenging systems. In physiological conditions, the balance between ROS generation and ROS scavenging is highly controlled. The energy production pathways (TCA cycle and OXPHOS), enzymatic reactions, by-products of metabolic pathways, and physical or chemical agents can lead to ROS production. As for the ROS-scavenging mechanisms, enzymatic defences and antioxidant scavengers neutralize the free radical reactions. When an imbalance between ROS production and ROS scavenging occurs, cells undergo oxidative stress, leading to severe cellular damage, cell death, and consequently whole organ and organism failure.

phosphate concentrations, and low adenine nucleotide concentration, is an essential permissive factor for mPTP opening [55]. This event triggers the so-called mitochondrial permeability transition that is characterized by a dramatic increase in the mitochondrial membrane permeability to any molecule smaller than $1.5 \mathrm{kDa}$. The consequent dissipation of the mitochondrial $\Delta \Psi_{\mathrm{m}}$ leads to membrane depolarization and mitochondrial swelling, increased mitochondrial reactive oxygen species (mROS) generation, cytochrome $\mathrm{c}$ release, and apoptosis [55].

The molecular identity of the MPTP is still debated. It was proposed that the adenosine nucleotide translocase (ANT), VDAC, and the translocator protein (TSPO) are essential components of the MPTP [54]. However, biochemical characterization to knockout models of these proteins suggests that they are dispensable for $\mathrm{mPTP}$ activity [56]. It has been recently proposed that MPTP is generated at the interface of two adjacent monomers of the F-ATP synthase through a strictly $\mathrm{Ca}^{2+}$-dependent mechanism, since gelexcised dimers of F-ATP synthase rapidly give rise to mPTP-like channels in lipid bilayers $[57,58]$. Nevertheless, the mechanism of PTP formation and activation is still debated. Detailed discussion of this aspect is beyond the scope of this review, and readers are referred to specific contributions on this topic [59-65].

2.1. Mitochondrial ROS Production and Regulation. Mitochondria, through the respiratory chain, especially complexes I and III, are considered the main source of physiological ROS [66]. mROS are generated in both physiological and pathological conditions [66]. Indeed, on the one hand moderate levels of ROS are involved in cell signalling by affecting the redox state of signalling proteins, but on the other hand, when in excess, mROS are among the major determinants of toxicity in cells and organisms [66].

During respiration, superoxide $\left(\mathrm{O}_{2}^{-}\right)$is produced by partial reduction of molecular oxygen. Subsequently, hydrogen peroxide $\left(\mathrm{H}_{2} \mathrm{O}_{2}\right)$ is formed by the action of matrix antioxidant defence enzymes as superoxide dismutase (SOD) [66]. $\mathrm{H}_{2} \mathrm{O}_{2}$ is transformed in water by glutathione peroxidase (GPX), peroxiredoxin (PRX), and catalases [66]. The regulation of the activity and the expression levels of these antioxidant enzymes are controlled by a plethora of mechanisms [66]. Under physiological conditions, the balance between ROS generation and ROS scavenging is highly controlled (Figure 2). Physiological ROS levels initiate a wide array of cellular responses, ranging from triggering signalling pathways, activation of mitochondrial fission and autophagy, adaptation to hypoxic condition, and differentiation to regulation of aging-related processes [67]. In these specific conditions, ROS production is induced in response to a stress and it functions as an intermediate signalling to facilitate cellular adaptation [68].

ROS production, when not compensated by ROS scavenging, results in oxidative stress leading to severe cellular damage and cell death [67]. In this condition, ROS become causative of several pathological states by the direct modification of cellular macromolecules, leading to alterations of the redox state of factors involved in signal transduction, inducing either hyper- or hypofunctionality of several signalling pathways $[67,68]$. Oxidative stress has been shown to be at the basis of aging and many pathological disorders. Indeed, ROS are responsible of cell death in pathological conditions such as myocardial infarct or stroke [67].

In physiological conditions and in a tissue-specific manner, mitochondrial $\mathrm{Ca}^{2+}$ uptake, by impinging on Krebs cycle enzymes and electron transport chain (ETC) activity, generates a ROS signals [69]. This signalling axis operates within a physiological window of $\left[\mathrm{Ca}^{2+}\right]$. Therefore, when $\left[\mathrm{Ca}^{2+}\right]$ overcomes this threshold, mROS production becomes detrimental and compromises mitochondrial bioenergetics and cell functions $[70,71]$. Mitochondrial $\mathrm{Ca}^{2+}$ may promote mROS formation both directly, by stimulating mROS-generating enzymes, like glycerol phosphate 
and $\alpha$-ketoglutarate dehydrogenase, and indirectly, as in the case of nitric oxide synthase (NOS) activation that, by forming NO, blocks complex IV, leading to excessive mROS formation [70].

Finally, mitochondrial $\mathrm{Ca}^{2+}$ overload triggers mPTP opening. Indeed, $\left[\mathrm{Ca}^{2+}\right]_{\mathrm{cyt}}$ increases beyond a certain value and induces mitochondrial $\mathrm{Ca}^{2+}$ overload, triggering the mitochondrial "permeability transition." In this condition, the mitochondrial membrane becomes permeable to any molecule less than $1.5 \mathrm{kDa}$ in size. Consequent dissipation of $\Delta \Psi_{\mathrm{m}}$ leads to a permanent membrane depolarization, decreased ATP production, and eventually cell apoptosis. Moreover, mitochondrial membrane depolarization leads to crista unfolding, uncoupling of oxidative phosphorylation, and the reverse electron transport (RET). RET is evoked when electrons from ubiquinol are transferred back to respiratory complex I, reducing $\mathrm{NAD}^{+}$to NADH. This process generates a significant amount of ROS [56].

Since mitochondrial $\mathrm{Ca}^{2+}$ plays a key role in $\mathrm{ROS}$ production, the cellular redox state can also significantly modulate $\mathrm{Ca}^{2+}$ signalling $[70,71]$. Indeed, it has been clearly demonstrated that redox equilibrium controls a variety of receptors, proteins, and other signalling molecules that, in turn, might directly or indirectly modify components of $\mathrm{Ca}^{2+}$ signalling pathways, thus altering $\mathrm{Ca}^{2+}$ homeostasis and reshaping local and global $\mathrm{Ca}^{2+}$ signals [70]. When the redox equilibrium is disturbed, due to the excessive accumulation or clearance of ROS, many cellular signalling pathways are influenced, leading to cellular dysfunction and subsequently to the development of various pathologies, including neurodegenerative disorders, cancer, diabetes, atherosclerosis, and ischemia/reperfusion (I/R) injury. Therefore, both mROS and mitochondrial $\mathrm{Ca}^{2+}$ signalling are two functional entities that strictly cooperate in order to contribute to the maintenance of cellular homeostasis [71].

Intriguingly, Dong and coworkers analysed the crosstalk between intracellular ROS levels and $\left[\mathrm{Ca}^{2+}\right]_{\text {mit }}$, suggesting that oxidative stress, and thus ROS accumulation, plays a positive feedback role in modulating MCU activity [72]. Indeed, they observed that MCU activity increases in cells exposed to endotoxin-mediated oxidative stress, leading to augmented $\left[\mathrm{Ca}^{2+}\right]_{\text {mit }}$ at resting $\left[\mathrm{Ca}^{2+}\right]_{\mathrm{cyt}}$. In detail, they identified a conserved cysteine in metazoan at position 97 (Cys-97) in the NTD of the MCU protein sequence to be the only reactive thiol in human MCU that undergoes redox modification (S-glutathionylation). The Cys-97 residue is surface-exposed and primed for an oxidative posttranslational modification that induces a conformational change of MCU that promotes the clustering of MCU channels and their persistent activation [72]. These data suggest that, in condition of oxidative stress, mROS overproduction in the mitochondrial matrix perturbs mitochondrial antioxidant activity resulting in S-glutathionylation of MCU Cys-97. The conjugation of glutathione causes a conformation change within the N-terminal domain that appears to promote MCU channel activity in resting condition. The increased MCU activity, in turn, enhances the production of $\mathrm{mROS}$ in the mitochondrial matrix in a positive feedback mechanism, thus leading to perturbation of mitochondrial bioenergetics and cell functions [72]. Overall, these data strongly suggest that ROS and mitochondrial $\mathrm{Ca}^{2+}$ signals are intimately interconnected, leading to a specific and adaptive response to given stimuli.

Excessive ROS are recognized as one of the causative factors in the development of a diverse array of diseases including cardiovascular, skeletal muscle, and neurodegenerative diseases and cancer progression (Figure 3 and $[67,71]$ ). This review is aimed at describing some pathological conditions characterized by a dysregulation of mitochondrial $\mathrm{Ca}^{2+}$ uptake associated with an excessive ROS production.

\section{Crosstalk of Mitochondrial $\mathrm{Ca}^{2+}$ Uptake and Mitochondrial Redox State in Physiopathology}

3.1. Heart. Most of the ATP necessary for cardiac excitation and contraction is synthesized within mitochondria via oxidative phosphorylation which, as described above, is a process modulated by $\mathrm{Ca}^{2+}[1]$. Furthermore, mitochondria are the major source of ROS that represent by-products of oxidative phosphorylation [73]. In living cells, and in particular in cardiac myocytes, ROS are also produced by extra-mitochondrial sources including NADPH oxidase, uncoupled NOS, xanthine oxidase, and monoamine oxidase [74]. In physiological conditions, ROS concentration is tightly regulated by antioxidants keeping them in a picomolar range. Low concentrations of ROS allow them to act as second messengers in signal transduction for vascular homeostasis and cell signalling [73]. In detail, the activity of redox-sensitive proteins, including $\mathrm{Ca}^{2+}$-handling proteins, contractile proteins, and proteins involved in various signalling pathways and in transcriptional activities, can be modulated by ROS [73].

Redox modulation of calcium-handling proteins directly affects cardiac contraction by altering intracellular calcium concentration [75]. In detail, ROS can oxidase and directly enhance the activity of $\mathrm{Ca}^{2+} /$ calmodulin-dependent kinase II (CaMKII) that in turn phosphorylates and activates several $\mathrm{Ca}^{2+}$-handling proteins such as the cardiac ryanodine receptor RyR2 or cardiac SERCA [76]. Cardiac RyR2 mediates $\mathrm{Ca}^{2+}$ release to the cytosolic compartment from SR during excitation-contraction coupling (ECC) and is itself subject to oxidation that increases RyR2 open probability but may lead to irreversible activation and $\mathrm{Ca}^{2+}$ leak [77]. Similarly to RyR2, cardiac SERCA, which transfers $\mathrm{Ca}^{2+}$ from the cytosol to the SR at the expense of ATP hydrolysis during diastole, might be also directly regulated by oxidation. In particular, low oxidation levels reversibly increase SERCA activity whereas higher levels cause irreversible inactivation [77].

When the equilibrium between ROS production and scavenging is altered, ROS can cause damage to lipids, proteins, and DNA by contributing to the development and progression of cardiovascular diseases such as atherosclerosis, I/R injury, chronic ischemic heart disease, cardiomyopathy, heart failure, and arrhythmias [73]. As already discussed in the previous paragraph, excessive ROS levels is caused not 


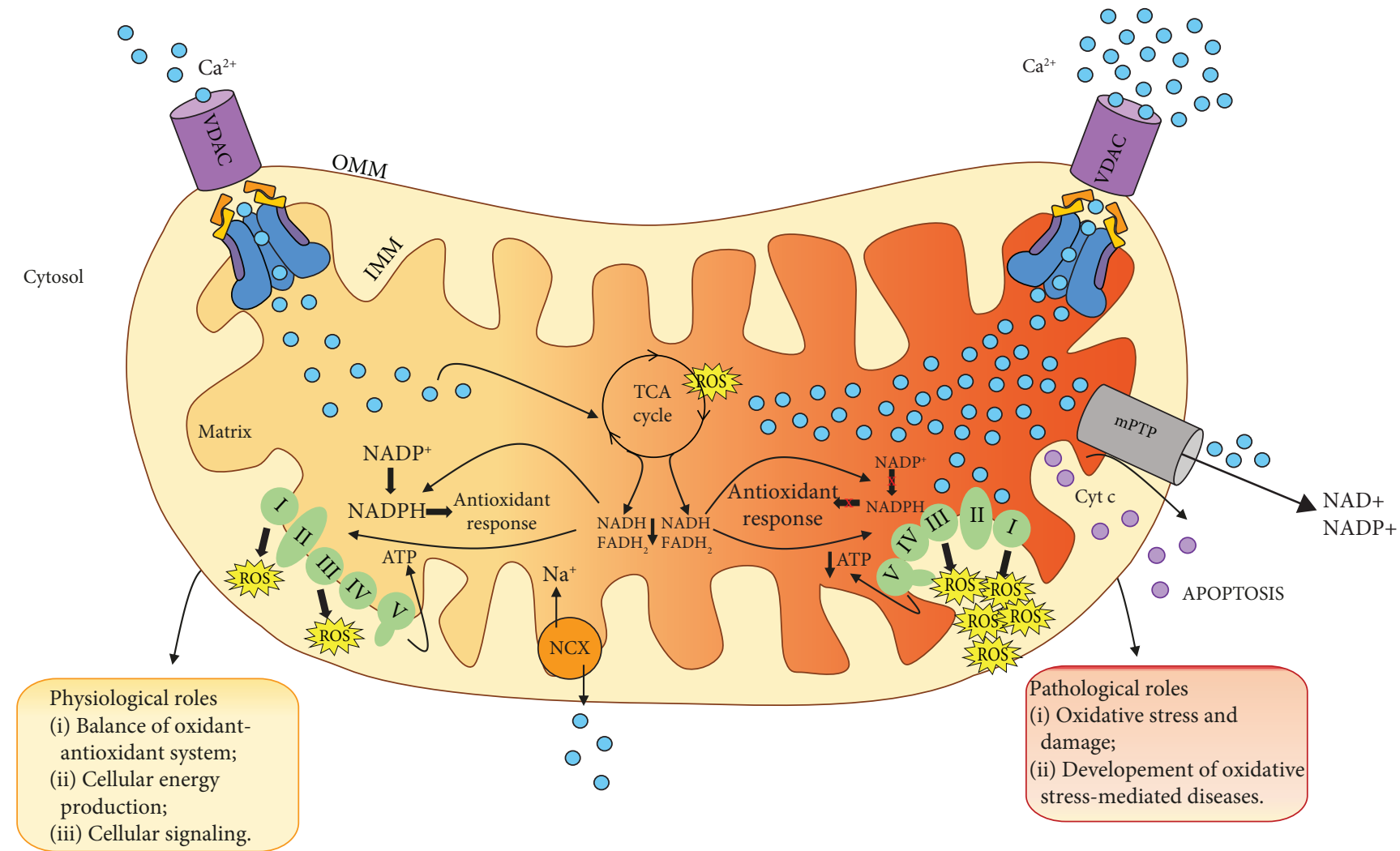

FIGURE 3: Regulation of mitochondrial $\mathrm{Ca}^{2+}$ uptake controls energy metabolism, mtROS production, and cell death. mtROS represent a by-product of oxidative phosphorylation and exert a beneficial or detrimental effect depending on their concentration and on the biological contest. In physiological conditions, mitochondrial $\mathrm{Ca}^{2+}$ uptake stimulates the TCA cycle and ATP production (left part). At physiological $\left[\mathrm{Ca}^{2+}\right]_{\text {mit }}$, the amount of ROS is counteracted by the activity of the antioxidant system (left part). In pathological conditions, excessive $\mathrm{Ca}^{2+}$ accumulation by mitochondria (right part) increases mtROS production which, in turn, affects antioxidant response. Altogether, this leads to the increase in the mPTP open probability, leading to irreversible collapse of the mitochondrial membrane potential, swelling of mitochondria, and thus release of cytochrome c, culminating in cell death.

only by defective ROS scavenging mechanisms but also by excessive ROS production.

Since cardiac mitochondria are the major producers of ROS through oxidative phosphorylation and $\mathrm{Ca}^{2+}$ plays a key role in promoting aerobic metabolism, dysregulation of mitochondrial $\mathrm{Ca}^{2+}$ homeostasis translates also in oxidative stress [73].

During myofibril contraction, ATP is hydrolysed to adenosine diphosphate (ADP) which moves into mitochondria through the adenine nucleotide transporter (ANT) and activates the $\mathrm{F}_{1} \mathrm{~F}_{0}$-ATPase to regenerate ATP [78]. The increase in mitochondrial ADP content accelerates electron flux along the ETC and induces the oxidation of the reduced $\mathrm{NADH}$ and $\mathrm{FADH}_{2}$ which act as electron donors to sustain oxidative phosphorylation [78]. At the same time, $\mathrm{Ca}^{2+}$ is accumulated into the mitochondrial matrix through the MCU, where it stimulates the activity of the Krebs cycle to replenish the reduced pyridine nucleotides as NADH and $\mathrm{FADH}_{2}$ which act as electron donors and sustain oxidative phosphorylation [78]. Therefore, $\mathrm{Ca}^{2+}$ plays a dual role since it both increases electron flux along the ETC and regenerates energy by increasing the electron flow from the Krebs cycle to the ETC $[79,80]$.
The ability of mitochondria to cope the increase in energy demand, occurring during increased cardiac workload or hormonal stimulation, is due to the close apposition of mitochondria and the SR $[3,81,82]$. It was hypothesized that $\mathrm{Ca}^{2+}$ released from the SR will elevate local $\mathrm{Ca}^{2+}$ to high levels resulting in a large mitochondrial $\mathrm{Ca}^{2+}$ influx [81]. Nevertheless, direct patch clamp recordings demonstrated that cardiac mitochondria's MCU current $\left(I_{\mathrm{MCU}}\right)$ is substantially smaller than that of other tissues, such as skeletal muscle, where $I_{\mathrm{MCU}}$ is 30 times bigger [83]. Notably, these two tissues present different mitochondrial volume fractions. Indeed, cardiac myocytes show one of the highest mitochondrial volume fractions in mammalian cells (37\%), while in skeletal muscle this fraction is much lower (5\%) [83]. Furthermore, also the MCU complex component stoichiometry is different between these two tissues. For example, in the heart, the expression of the dominant-negative subunit MCUb is higher than that in the skeletal muscle, inversely correlating with mitochondrial $\mathrm{Ca}^{2+}$ current [38]. In addition, mitochondrial $\mathrm{Ca}^{2+}$ uptake in the heart is controlled by a low MICU1/MCU ratio, thus lowering the threshold and cooperativity of the MCU complex [45]. It has been proposed that both the low $\mathrm{Ca}^{2+}$ conductivity of the MCU and the 
complexity of its regulatory components could represent protective mechanisms by which cardiac mitochondria regulate $\left[\mathrm{Ca}^{2+}\right]_{\text {mit }}$ by preventing $\mathrm{Ca}^{2+}$ overload [83]. Indeed, an excessive mitochondrial $\mathrm{Ca}^{2+}$ uptake associated with an increase in ROS accumulation leads to the opening of the PTP, irreversible collapse of mitochondrial membranepotential, swelling of mitochondria, and thus release of cytochrome c resulting in necrotic cardiomyocyte cell death, a common scenario observed in the ischemic/reperfused myocardium [55].

In chronic heart failure (HF), perturbations of ECC cause contractile dysfunction [84] which is the result of decreased systolic $\mathrm{Ca}^{2+}$ transients, caused by at least three mechanisms that altogether contribute to reduce $\left[\mathrm{Ca}^{2+}\right]_{\text {mit }}$ by affecting the activation of the Krebs cycle during increased workload [85]. The first mechanism is associated with a decreased $\mathrm{Ca}^{2+}$ load of the SR, with consequent reduction of cytosolic $\mathrm{Ca}^{2+}$ transients. The reduction in SR $\mathrm{Ca}^{2+}$ load is due both to a lower activity of the SERCA and to leaky RyR2 [84]. During relaxation, $\mathrm{Ca}^{2+}$ removal is mainly due to SERCA and the sarcolemmal NCX. Interestingly, in HF, SERCA activity is reduced and, concomitantly, NCX activity increases. As a consequence, more $\mathrm{Ca}^{2+}$ is extruded from the cell and less is taken up by SERCA, thus decreasing the SR $\mathrm{Ca}^{2+}$ available to be released during subsequent ECC $[86,87]$. The second mechanism, associated with contractile dysfunction in failing cardiomyocytes, is the huge increase in cytosolic $\mathrm{Na}^{+}$levels $\left(\left[\mathrm{Na}^{+}\right]_{\text {cyt }}\right)$, which accelerates mitochondrial $\mathrm{Ca}^{2+}$ efflux via the mitochondrial $\mathrm{Na}^{+} / \mathrm{Ca}^{2+}$ exchanger (mNCX) $[88,89]$. This hampers the activation of Krebs cycle dehydrogenases by $\mathrm{Ca}^{2+}$, and it results in pronounced oxidation of NADH to $\mathrm{NAD}^{+}$during transitions of workload [90]. The higher $\left[\mathrm{Na}^{+}\right]_{\text {cyt }}$ could be explained by lower $\mathrm{Na}^{+} / \mathrm{K}^{+}$pump activity, consistent with a decreased $\mathrm{Na}^{+} / \mathrm{K}^{+}$pump expression in some models of $\mathrm{HF}[91,92]$. However, this finding was questioned by a more recent study performed in HF rabbit ventricular myocytes demonstrating that the higher $\left[\mathrm{Na}^{+}\right]_{\mathrm{cyt}}$ is due to elevated diastolic $\mathrm{Na}^{+}$influx rather than altered $\mathrm{Na}^{+} / \mathrm{K}^{+}$pump activity [93]. The huge increase in $\left[\mathrm{Na}^{+}\right]_{\mathrm{cyt}}$ modifies the direction of the sarcoplasmic NCX transport by inducing the increase in cytosolic $\mathrm{Ca}^{2+}$ that partially compensates the decreased SR $\mathrm{Ca}^{2+}$ load and release in failing myocytes [89]. On the contrary, no compensatory effects have been detected on mitochondrial $\mathrm{Ca}^{2+}$ uptake since the rather slow NCX-mediated $\mathrm{Ca}^{2+}$ influx shows less impact on mitochondrial $\mathrm{Ca}^{2+}$ uptake [94]. The third mechanism involves changes in the activity of the MCU. Indeed, Michels and colleagues demonstrated that in human cardiac mitochondria from patients affected by HF, the open probability of MCU is decreased [94]. This affects both the energy supply and demand matching and thus the oxidative capacity. This evidence was also observed in isolated cardiomyocytes from a guinea pig model of systolic HF characterized by decreased $\mathrm{NADH}$ and NADPH levels and thus in the amount of reducing equivalents necessary for ATP production accompanied by an increase in ROS levels [95].

ROS directly act on cellular structures and activate signalling molecules involved in myocardial remodelling and failure. Indeed, on the one hand low levels of ROS are associated with the activation of the mitogen-activated protein kinase (MAPK) and protein synthesis; on the other hand, high levels of ROS affect ECC in cardiac myocytes [96]. The latter can cause arrhythmias, activate prohypertrophic signalling, and induce apoptotic and/or necrotic cell death through the activation of the MPTP [78]. In addition, mitochondrial ROS play a key role in the development and progression of $\mathrm{HF}$ in response to different stimuli such as I/R, pressure overload, and angiotensin II [97]. It has also been demonstrated that oxidative stress stimulates the activity of myocardial metalloproteinases (MMP), a family of proteolytic enzymes that regulates extracellular matrix turnover and that is implicated in the cardiac remodelling after myocardial infarction [98].

The concept that ROS production by cardiac mitochondria is dynamically regulated by $\mathrm{Ca}^{2+}$ and ADP and controlled by the redox state of mitochondrial pyridine nucleotides gave rise to the finding that an imbalance between decreased mitochondrial $\mathrm{Ca}^{2+}$ uptake and increased cardiac workload triggers oxidative stress [78]. As already discussed above, mitochondrial $\mathrm{Ca}^{2+}$ uptake plays a key role in matching ATP production to demand and has a great impact on the redox state of pyridine nucleotides [78]. Therefore, the regulation of mitochondrial $\mathrm{Ca}^{2+}$ uptake mediated by the MCU complex in the pathophysiology of heart failure has been extensively investigated in the past years. Indeed, after the characterization of the MCU complex, three different mouse models have been generated: a mouse model with a constitutive global MCU knockout (KO) [99, 100], a conditional cardiac myocyte-specific MCU KO [101, 102], and a transgenic mouse overexpressing a dominant-negative form of MCU (DN-MCU) [103, 104].

Surprisingly, total body knockout of MCU mice is viable and exhibits a very mild phenotype, with slightly smaller body mass than those of wild-type littermates and a slight decrease in skeletal muscle strength and performance [99]. As for the heart, no differences between MCU KO heart and WT littermates were observed [99]. As expected, MCU knockout cardiac mitochondria seem incapable of any rapid mitochondrial calcium uptake and showed alterations in $\mathrm{Ca}^{2+}$-dependent oxygen consumption although basal ATP levels were unaltered, suggesting that MCU depletion does not affect basal mitochondrial metabolism [100]. These results can be partially explained by the observation that resting free $\left[\mathrm{Ca}^{2+}\right]$ in knockout mitochondria is only partially reduced [99]. Altogether, these results suggest the existence of alternative mechanisms for $\mathrm{Ca}^{2+}$ accumulation in basal conditions, although MCU KO mitochondria were not able to take up $\mathrm{Ca}^{2+}$ during cell stimulation [99], fundamental phenomena for responding to variation in energy demands during cell activation. Furthermore, MCU deletion is viable in the outbred CD1 strain, while it results in embryonic lethality in the inbred C57BL/6 strain, suggesting the existence of compensatory mechanisms that allow animal viability only in the mixed background [105]. Another puzzling result is the lack of protection from cell death in the hearts lacking MCU. Indeed, in isolated mitochondria from $\mathrm{KO}$ hearts, the addition of large amounts of extramitochondrial 
calcium did not lead to mPTP activation. Surprisingly following global $\mathrm{I} / \mathrm{R}$ injury, infarct size was indistinguishable between WT hearts and hearts from MCU KO mice [99], but cyclosporine $\mathrm{A}$, an inhibitor of $\mathrm{mPTP}$, was able to reduce infarct size only in hearts of WT hearts.

As regards the heart-specific MCU KO mice and the transgenic mice overexpressing DN-MCU, a common feature is that, although mitochondria isolated from the heart of these mice are unable to accumulate $\mathrm{Ca}^{2+}$, cardiomyocyte function is altered only after $\beta$ adrenergic receptor $(\beta$-AR) stimulation. This highlights the importance of MCU-mediated mitochondrial $\mathrm{Ca}^{2+}$ uptake in sustaining cardiac function during physiological increases in workload. In addition, in DN-MCU mice, it was observed that $\left[\mathrm{Ca}^{2+}\right]_{\text {cyt }}$ increases after the positive inotropic and chronotropic responses to $\beta-A R$ stimulation $[103,104]$. This finding, corroborated by a study performed on neonatal cardiomyocytes in which MCU was silenced, suggests that MCU may contribute to cytosolic $\mathrm{Ca}^{2+}$ buffering in the heart $[81,104]$.

The genetic ablation of MCU in the heart affects reducing equivalent productions. Since the redox state of NADH is closely linked to NADPH through the activity of a key antioxidant enzyme, the mitochondrial membrane-bound nicotinamide nucleotide transhydrogenase (Nnt), which normally regenerates NADPH from NADH, reduced mitochondrial $\mathrm{Ca}^{2+}$ uptake in failing myocytes which increases ROS production $[56,85,106]$. Oxidative stress, in turn, increases $\left[\mathrm{Na}^{+}\right]_{\text {cyt }}[107]$ and enhances NCX-mediated $\mathrm{Ca}^{2+}$ influx [108], thus generating a vicious cycle of defective ECC, reduced mitochondrial $\mathrm{Ca}^{2+}$ uptake, energetic deficit, and oxidative stress, a common scenario observed in HF. Overall, these findings suggest that, in HF, a mismatch of workload and mitochondrial $\mathrm{Ca}^{2+}$ uptake causes oxidation of pyridine nucleotides by inducing energy deprivation and oxidative stress [78].

Both the global constitutive MCU KO and DN-MCU mouse models are characterized by a constitutive modulation of MCU activity that may not exclude adaptations to embryonic long-term loss of mitochondrial $\mathrm{Ca}^{2+}$ uptake $[99,100,103,104]$. Phenomena of adaptation have been excluded with the generation of a conditional cardiac MCU KO. Surprisingly, the heart phenotype observed in this mouse was quite similar to constitutive mouse models $[101,102]$. However, deletion of MCU in adult cardiomyocytes leads to protection from cell death induced by $\mathrm{I} / \mathrm{R}$ injury [104]. Furthermore, $\mathrm{Ca}^{2+} / \mathrm{calmodulin}$-dependent protein kinase II (CaMKII) has been proposed to induce mPTP opening-dependent myocardial death by increasing $I_{\mathrm{MCU}}$ [109]. CAMKII directly interacts and increases $I_{\mathrm{MCU}}$. Coherently, transgenic mice with myocardial expression of CaMKIIN, a CaMKII inhibitor, targeted to mitochondria, were protected against I/R injury and showed reduced mitochondrial $\mathrm{Ca}^{2+}$ uptake and decreased $I_{\mathrm{MCU}}$. Nevertheless, these findings have been questioned since the $I_{\mathrm{MCU}}$ recorded was two orders of magnitude greater than the one previously measured and characterized by high fluctuations incompatible with the low single-channel conductance of MCU [110].
Altogether, these findings demonstrated that the MCUmediated regulation of mitochondrial $\mathrm{Ca}^{2+}$ uptake plays a key role in the onset and progression of HF and thus may be useful for the development of novel treatments targeting mitochondria to ameliorate the progression of the disease.

3.2. Skeletal Muscle. Mitochondria play a crucial role in skeletal muscle function by providing ATP largely consumed by actomyosin contraction and SERCA activity. As in the heart, skeletal muscle mitochondria are commonly considered as the predominant source of ROS [111]. Indeed, during exercise, the intense skeletal muscle contractile activity enhances mitochondrial oxidative phosphorylation that increases the oxygen consumption rate and thus ROS production [111]. In detail, it has been demonstrated that $\mathrm{O}_{2}{ }^{-}$generation in skeletal muscle increases to about 50- or 100-fold during aerobic contraction [112]. Although ROS have been considered as deleterious species for skeletal muscle tissue, several evidences indicate that they might also play a positive role in physiological processes occurring in muscle cells [111]. Whether a beneficial or detrimental effect prevails depends on several variables among which the most relevant are the duration of ROS flow, the site of ROS production, and the antioxidant status of the cells [111]. In line with this, recently the redox-optimized ROS balance theory has been postulated that assumes that ROS levels depend on the redox state of a cell $[113,114]$. In detail, ROS production increases both at high electron fluxes along the ETC, because the ROS production overwhelms the scavenging systems, and when the cellular metabolic state is reduced since reducing equivalents to sustain the antioxidant defences are lacking. Thus, ROS emission from mitochondria is minimal at an intermediate redox state with low ROS production at the ETC, but with sufficient levels of reduced NADPH to neutralize ROS [114]. Although the threshold of physiological ROS concentration above which ROS exert their negative effects has not been yet characterized, different pathways induced or affected by ROS in skeletal muscle has been intensively studied [111]. In particular, low levels of ROS activate signalling molecules such as PGC1- $\alpha$, AMPK, and MAPK that control mechanisms of muscle adaptation such as oxidative metabolism and mitochondrial biogenesis [111]. Importantly, they also exert a self-control mechanism by regulating the activity of antioxidant enzymes. On the contrary, high levels of ROS induce functional oxidative damage of proteins, lipids, nucleic acids, and cell components, a huge increase in intracellular $\left[\mathrm{Ca}^{2+}\right]$ leading to apoptosis and necrosis [111]. Furthermore, several studies demonstrated that dysregulation in ROS production has been considered as causal factors in various muscular pathologies [115-118]. In particular, oxidative stress appears to trigger the myopathic phenotype of malignant hyperthermia susceptibility (MHS) and central core disease (CCD) [119]. MHS is a pharmacogenetic disorder characterized by life-threatening episodes after treatment with depolarizing muscle relaxants while CCD represents one of the most common congenital myopathies [120]. Mutations of the RyR1 gene account for the majority of cases of MHS and CCD [120]. Initial treatment for MHS includes the administration of the RyR antagonist dantrolene, a 
hydantoin derivate that inhibits the release of $\mathrm{Ca}^{2+}$ from SR without stimulating its reuptake [121]. However, since dantrolene suppresses $\mathrm{Ca}^{2+}$ release and $\mathrm{Ca}^{2+}$ entry, it is not surprising that it could affect protein involved in ECC [122]. Nevertheless, dantrolene is currently the only available pharmacological treatment of $\mathrm{MH}$ [121]. Importantly, in several models of these pathologies, it has been observed that the treatment with antioxidant agents ameliorates the muscular phenotype [123]. Indeed, it has been demonstrated that enhanced $\mathrm{Ca}^{2+}$ leak from mutant RyR1 increases oxidative/nitrosative stress in the RyR ${ }^{\mathrm{Y} 522 \mathrm{~S}}$ knock-in mice. This oxidative stress leads to S-nitrosylation of RyR1 which further enhances $\mathrm{Ca}^{2+}$ leak from this channel and increases susceptibility to heat-induced sudden death [124].

Dysregulation of $\left[\mathrm{Ca}^{2+}\right]_{\text {mit }}$ associated with an increase in ROS production has been proposed as a possible mechanism for skeletal muscle fiber death in Duchenne muscular dystrophy (DMD) [125]. DMD is caused by loss-of-function mutations in the dystrophin gene located in chromosome X [125]. Loss of dystrophin protein increases muscle membrane permeability by inducing a huge increase in $\left[\mathrm{Ca}^{2+}\right]_{\text {cyt }}$ that, in turn, induces mitochondrial $\mathrm{Ca}^{2+}$ overload [125]. This causes dysfunction of several oxidative phosphorylation enzymes and is accompanied by decreased ATP-synthase activity by influencing both ROS and ATP production in DMD muscles [125]. Increased mitochondrial $\mathrm{Ca}^{2+}$ load, occurring in DMD muscles, impairs the ability of mitochondria to reduce free radicals [125] and leads to the onset of apoptotic pathways that culminate in muscle atrophy [125]. This evidence places mitochondria as central participants in the aetiology of DMD, describing the relationship between increased intracellular $\left[\mathrm{Ca}^{2+}\right]$, mitochondrial permeability, and dysfunction culminating in muscle loss [125].

Among the mechanisms that induce the increase in ROS production, the regulation of mitochondrial $\mathrm{Ca}^{2+}$ uptake is one of the most relevant. As already mentioned above, the MCU complex plays a key role in regulating $\mathrm{Ca}^{2+}$ entry into mitochondria and therefore it might be implicated in the development and progression of different muscular diseases [1]. In particular, the skeletal muscle of total MCU KO mice represents the most affected tissue [99], probably because it shows a much higher $I_{\mathrm{MCU}}$ compared to the heart (see previous paragraph and [83]). Interestingly, it has also been demonstrated that loss-of-function mutations of the MICU1 gene in humans causes dysfunctional $\mathrm{Ca}^{2+}$ uptake and results in clinical and pathological features that usually characterize mitochondrial myopathies, congenital core myopathies, and muscular dystrophies [42]. In particular, muscle biopsies from affected individuals clearly show a myopathic phenotype, characterized by a diffuse variation in fiber size, increased frequency of internal and central nuclei, and clusters of regenerating fibers, without pronounced fibrosis or fat infiltration. Surprisingly, two different MICU1 KO mice display perinatal mortality. One of these models displays an incomplete penetrance, and the $\mathrm{KO}$ animals that survive exhibit marked ataxia and muscle weakness, which progressively ameliorate during growth [126]. The physiological relevance of the MCU complex components, and thus the regulation of mitochondrial $\mathrm{Ca}^{2+}$ uptake in the onset and progression of muscular diseases, identifies the MCU complex as a potential target for the development of specific pharmacological therapies aimed at both improving the quality of life and increasing the life span of patients.

3.3. Neurons. The regulation of mitochondrial shape, volume, number, and distribution within the cells influences mitochondrial function especially in the CNS, where mitochondria show a strategic intracellular distribution, according to local energy demand [127]. Indeed, neurons require extremely precise spatiotemporal control of $\mathrm{Ca}^{2+}$-dependent processes, since they regulate vital functions such as transmission of depolarizing signals, synaptic plasticity, and metabolism [1]. For this reason, neurons are extremely sensitive to variations of $\left[\mathrm{Ca}^{2+}\right]$, and even small defects in $\mathrm{Ca}^{2+}$ homeostasis, hallmark of aging and neurodegenerative diseases, are able to impair neuronal activity [128, 129]. $\left[\mathrm{Ca}^{2+}\right]_{\text {cyt }}$ increases in neurons principally occur through $\mathrm{Ca}^{2+}$ entry from the plasma membrane through ligand-gated glutamate receptors, such as the N-methyld-aspartate receptor (NMDAR) or various voltagedependent $\mathrm{Ca}^{2+}$ channels (VDCCs), as well as from the release of $\mathrm{Ca}^{2+}$ from intracellular stores [130]. The contribution of these sources to intracellular $\mathrm{Ca}^{2+}$ in neurons depends on their size, transmitter system, and location in neural circuits (excitatory or inhibitory) [130]. In addition, in presynaptic neurons, $\mathrm{Ca}^{2+}$ entry through voltageoperated $\mathrm{Ca}^{2+}$ channels promotes the release of neurotransmitters into the synaptic cleft that, in turn, activates receptors located in the postsynaptic plasma membrane by initiating signal transmission [129]. This event generates $\mathrm{Ca}^{2+}$ signals that induce specific responses according to the type of receptors that have been activated [129]. Beyond its importance in synaptic transmission, mitochondrial $\mathrm{Ca}^{2+}$ uptake guarantees activity-dependent regulation of cellular energy metabolism [131]. Neurons use mitochondrial oxidative phosphorylation to generate ATP, required for cellular metabolism. The major by-product of this process is $\mathrm{O}_{2}{ }^{-}$which is dismutated to $\mathrm{H}_{2} \mathrm{O}_{2}$ by the mitochondrial enzyme superoxide dismutase 2 (SOD2) [132]. Since neurons show an extremely high metabolic rate, they produce elevated amounts of ROS in comparison to other organs [132].

In physiological conditions, ROS play active roles in many cellular processes. In particular, in the nervous system, ROS production regulates neuronal development, differentiation, and axon formation [132]. In particular, angiotensin II (Ang-II), brain-derived neurotrophic factor (BDNF), and vascular cell adhesion molecule-1 (VCAM-1) modulate cellular ROS production to regulate neural precursor proliferation and differentiation $[133,134]$. Furthermore, it has also been demonstrated that ROS participate in synaptic plasticity as second messengers in several areas of the CNS, including the hippocampus, cerebral cortex, spinal cord, hypothalamus, and amygdala [135-139]. In this regard, it has been shown that repetitive stimuli, by inducing high $\mathrm{Ca}^{2+}$ influx, cause an increase in mitochondrial superoxide production. The latter induces the activation of CaMKII and PKA, two kinases involved in synaptic potentiation [132]. Furthermore, it has been demonstrated that increased mitochondrial 
$\mathrm{Ca}^{2+}$ uptake and the consequent stimulation of ROS production plays a key role for the induction of the long-term potentiation (LTP), the principal form of synaptic plasticity in the mammalian brain, thought to endure experience-dependent enhancement of synaptic transmission [132]. In detail, inhibition of MCU blocks potentiation despite the increase in cytosolic $\mathrm{Ca}^{2+}$ levels produced after NMDA receptor activation [140]. Mitochondrial ROS, mainly superoxide, activate downstream signalling cascades involving PKA, PKC, and ERK which in turns results in synaptic plasticity of the dorsal horn neurons [140].

Mitochondria-derived ROS levels are regulated by intracellular $\mathrm{Ca}^{2+}$ levels. Indeed, ROS increase when mitochondria are exposed to high $\left[\mathrm{Ca}^{2+}\right]$ and $\left[\mathrm{Na}^{+}\right]$, for example, after having sustained NMDA receptor activation [141, 142]. $\mathrm{Ca}^{2+}$ influx from N-methyl-d-aspartate (NMDA) receptors triggers mitochondrial activation of caspase 3 which stimulates the synthesis of the myocyte enhancer factor 2 (MEF2) that regulates the transcription of the mitochondrial gene NADH dehydrogenase 6 (ND6), which encodes an essential component of complex I [143]. The MEF2-dependent expression of ND6 reduces cellular levels of the antioxidant enzymes superoxide dismutase and hydrogen peroxidase by increasing oxidative stress [143]. Therefore, dysregulation of mitochondrial $\mathrm{Ca}^{2+}$ uptake, and thus a decrease in the rate of ATP production, may influence mitochondrial metabolism and function, thus affecting neuronal activity [131]. In particular, excessive mitochondrial $\mathrm{Ca}^{2+}$ accumulation induces an overproduction of ROS that has detrimental effects on neurons [144]. Although mitochondria produce the largest amount of cellular ROS, other sources contribute to the generation of ROS in neurons such as the enzyme neural NOS and the NADPH oxidase. The huge increase in ROS levels induces cellular damage, impairment of the DNA repair system, and mitochondrial dysfunction, all of which are recognized as major determinants of aging and of neurodegenerative disorder development [132].

A recent study highlighted the importance of MCU in controlling excitotoxicity and its implication in NMDA receptor-mediated cell death [145]. In this study, Qiu and coworkers demonstrated that the overexpression of MCU in hippocampal and cortical neurons of newborn mice causes an NMDA-mediated increase in the $\left[\mathrm{Ca}^{2+}\right]_{\text {mit }}[145]$. This increase, in concert with $\mathrm{NO}$ production and activation of poly (ADP-ribose) polymerase-1 (PARP-1), leads to the loss of mitochondrial membrane potential which in turn energetically compromises neurons and leads to ROS generation [145]. In addition, knockdown of MCU in neurons causes a decrease in NMDA-mediated mitochondrial $\mathrm{Ca}^{2+}$ levels, thus preventing the loss of the mitochondrial membrane potential and excitotoxic cell death [145]. These findings suggest that $\mathrm{MCU}$, and thus mitochondrial $\mathrm{Ca}^{2+}$, plays an essential role in neuronal excitotoxicity, although more studies are required to confirm the function of MCU in vivo.

3.4. Cancer. Tumor formation and progresssion are directly related to mitochondrial dysfunction [146]. Furthermore, reprogramming of mitochondrial metabolism and an aberrant $\mathrm{Ca}^{2+}$ homeostasis are considered hallmarks of cancer cells [146]. Multiple lines of evidence highlighted the key role of $\mathrm{Ca}^{2+}$ homeostasis deregulation in tumor cell proliferation, apoptosis resistance, tumor development, and metastasis [147]. Although mitochondria exert a key role in cancer progression and $\mathrm{Ca}^{2+}$ signalling is altered in a wide variety of tumors, the mechanisms that connect mitochondrial $\mathrm{Ca}^{2+}$ homeostasis with malignant tumor formation and growth have not been characterized yet. Recently, Marchi et al. demonstrated that prostate and colon cancers overexpress an MCU-targeting microRNA that, by reducing mitochondrial $\mathrm{Ca}^{2+}$ uptake, allows cancer cell resistance to apoptotic stimuli thus increasing tumor cell survival [148]. However, a correlation between MCU overexpression and poor prognosis in breast cancer patients was also recently hypothesized [149]. This study demonstrated that in the MDA-MB-231 cell line, a triple-negative breast cancer model (TNBC), MCU expression correlates with breast tumor size and lymph node infiltration [149]. Coherently, MCU silencing causes a significant decline in $\left[\mathrm{Ca}^{2+}\right]_{\text {mit }}$, metastatic cell motility, and matrix invasiveness. Most importantly, in MDA-MB-231 xenografts, deletion of MCU greatly reduces tumor growth and metastasis formation and this is associated with a decrease in mitochondrial ROS production, suggesting that mitochondrial ROS might play a crucial role in cell malignancy regulation by mitochondrial $\mathrm{Ca}^{2+}$ uptake [149]. In addition, MCU silencing in TNBC cells downregulates hypoxia-inducible factor 1-alpha (HIF1- $\alpha$ ) expression, thus negatively affecting the expression of HIF1- $\alpha$ target genes involved in cancer progression [149]. Elevated levels of ROS have been detected in almost all cancers including Akt-positive tumors [150]. Akt or more commonly known as protein kinase $\mathrm{B}(\mathrm{PKB})$ is a cytosolic protein kinase that regulates cellular energy metabolism and apoptosis through mechanisms that converge on mitochondria or via the phosphorylation of key proteins like the $\mathrm{Bcl}-2$-associated death promoter $(\mathrm{BAD})$ protein. $\mathrm{BAD}$ is a proapoptotic member of the $\mathrm{Bcl}-2$ gene family which is involved in initiating apoptosis. Marchi and coworkers demonstrated that Akt phosphorylates MICU1 at the N-terminal domain by affecting MICU1 proteolytic maturation and stability, thus altering mitochondrial $\mathrm{Ca}^{2+}$ uptake homeostasis [151]. Akt-mediated phosphorylation of the MCU complex regulator MICU1 may sustain cancer progression by increasing the basal mitochondrial $\mathrm{Ca}^{2+}$ level and ROS production [151]. In addition, mitochondrial $\mathrm{Ca}^{2+}$ uptake not only represents a fundamental mechanism to regulate cell survival and metabolism but also plays a pivotal role in the regulation of autophagy that plays both a negative and a positive role in cancer [152, 153]. In particular, mitophagy is an essential process that maintains mitochondrial quality and number by the removal of damaged or unnecessary mitochondria using autophagic machinery, thus limiting cellular degeneration [154]. Increasing evidence from different studies supports the concept that dysregulation of mitophagy is an etiologic factor in tumorigenesis [155]. Even though tumorigenesis relies on inhibition of mitophagy, tumor progression likely relies on the presence of functional mitophagy [156-160].

It is important to underline that dysregulation of mitophagy represents a scenario that characterizes not only cancer 
but also different spectra of diseases including neurodegenerative diseases, motor neuron disorders, autosomal dominant optic atrophy, I/R injury, diabetes, aging, and cancer [161]. The discussion of this aspect is beyond the scope of this review and has been reviewed in greater detail elsewhere.

Altogether, these data reveal the importance of the association between aberrant mitochondrial $\mathrm{Ca}^{2+}$ levels and tumor development and strongly suggest that alteration in the activity of the MCU complex components represents a critical checkpoint of metastatic behaviour and thus a potential pharmacological target to combat aggressive cancers.

\section{Conclusions}

Mitochondria are key intracellular organelles that play a fundamental role in energy production and control many cellular processes from signalling to cell death. The function of the mitochondrial electron transport chain, the major source of ATP in the cell, is coupled with the production of ROS that are maintained at physiological levels by highly efficient mitochondrial antioxidant systems. Moreover, these antioxidant defences rely on mitochondrial metabolism that supplies the reducing equivalents needed for their activity.

In the last years, several studies demonstrated that quick changes in ROS levels, coupled with essential cellular functions, are fundamental participants of physiological signalling. Importantly, mitochondrial calcium, by impinging on aerobic metabolism, plays a crucial role in this process, since it joins the cellular activation stimuli and ROS production. This phenomenon plays a crucial role in the maintenance of cellular homeostasis in several tissues, as discussed above.

When the balance between ROS production and clearance is altered by either overproduction of mROS or impairment of the antioxidant defence, mitochondrial dysfunction occurs, leading to the induction of the cell death cascade. Indeed, the overproduction of mROS and the change in mitochondrial redox homeostasis have been shown to be involved in several pathological conditions, which often are associated with mitochondrial $\mathrm{Ca}^{2+}$ overload. For these reasons, the physiopathological role of mitochondrial $\mathrm{Ca}^{2+}$ uptake and mROS production has been extensively studied in the past years.

The molecular and functional characterization of the MCU complex components highlights the importance of the dynamic regulation of mitochondrial $\mathrm{Ca}^{2+}$ in organ physiology. In particular, in this review, we highlighted the contribution of the MCU complex activity, and thus the regulation of mitochondrial $\mathrm{Ca}^{2+}$ uptake, in cardiovascular, skeletal muscle, and neurodegenerative diseases and cancer. Although after the discovery of the molecular identity of the MCU complex many studies have confirmed the crucial role of mitochondrial $\mathrm{Ca}^{2+}$ signals in the regulation of cell survival, metabolism, and autophagy, many findings are controversial, and many questions are still open. In this regard, the characterization of the mechanisms responsible for the survival of the total MCU KO mouse only in the outbred strain and the analysis of the mechanisms underlying the different phenotypes of the MICU1 KO mice will be fundamental important to finely dissect the physiological role of mitochondrial $\mathrm{Ca}^{2+}$. Furthermore, the structural and functional complexity of the MCU complex needs to be clarified. Thus, the study of the physiological role of the different MCU complex components might be useful to better characterize the regulation of mitochondrial $\mathrm{Ca}^{2+}$ uptake in different physiopathological conditions thus resulting in the identification of novel therapeutic strategies to cure pathologies characterized by dysregulation of mitochondrial $\mathrm{Ca}^{2+}$ homeostasis. In line with this, the discovery of drugs that modulate the activity of the MCU complex shall be extremely relevant for the future development of MCUtargeting therapies.

\section{Conflicts of Interest}

The authors declare that there is no conflict of interest regarding the publication of this paper.

\section{Authors' Contributions}

Simona Feno and Gaia Butera contributed equally to this work.

\section{Acknowledgments}

Research was supported by funding from the Italian Ministry of Education, the Fondazione Telethon (GGP16029 to R.R. and GGP16026 to A.R.), the Italian Association for Cancer Research (IG 18633 to R.R.), the French Muscular Dystrophy Association (19471 to A.R.), and the Italian Ministries of Health (Ricerca Finalizzata) (GR-2016-02362779 to A.R.).

\section{References}

[1] C. Mammucari, A. Raffaello, D. Vecellio Reane, G. Gherardi, A. De Mario, and R. Rizzuto, "Mitochondrial calcium uptake in organ physiology: from molecular mechanism to animal models," Pflügers Archiv - European Journal of Physiology, vol. 470, no. 8, pp. 1165-1179, 2018.

[2] R. Rizzuto, D. De Stefani, A. Raffaello, and C. Mammucari, "Mitochondria as sensors and regulators of calcium signalling," Nature Reviews Molecular Cell Biology, vol. 13, no. 9, pp. 566-578, 2012.

[3] D. De Stefani, R. Rizzuto, and T. Pozzan, "Enjoy the trip: calcium in mitochondria back and forth," Annual Review of Biochemistry, vol. 85, no. 1, pp. 161-192, 2016.

[4] R. Rizzuto, S. Marchi, M. Bonora et al., " $\mathrm{Ca}^{2+}$ transfer from the ER to mitochondria: when, how and why," Biochimica et Biophysica Acta (BBA) - Bioenergetics, vol. 1787, no. 11, pp. 1342-1351, 2009.

[5] M. Schmidt, P. A. Oude Weernink, F. vom Dorp, M. B. Stope, and K. H. Jakobs, "Mammalian phospholipase C," Advances in Molecular and Cell Biology, vol. 33, pp. 431-450, 2004.

[6] A. Raffaello, C. Mammucari, G. Gherardi, and R. Rizzuto, "Calcium at the center of cell signaling: interplay between endoplasmic reticulum, mitochondria, and lysosomes," Trends in Biochemical Sciences, vol. 41, no. 12, pp. 10351049, 2016.

[7] R. Chandrasekhar, K. J. Alzayady, and D. I. Yule, "Using concatenated subunits to investigate the functional consequences of heterotetrameric inositol 1,4,5-trisphosphate 
receptors," Biochemical Society Transactions, vol. 43, no. 3, pp. 364-370, 2015.

[8] R. Chandrasekhar, K. J. Alzayady, L. E. Wagner, and D. I. Yule, "Unique regulatory properties of heterotetrameric inositol 1,4,5-trisphosphate receptors revealed by studying concatenated receptor constructs," Journal of Biological Chemistry, vol. 291, no. 10, pp. 4846-4860, 2016.

[9] J. T. Lanner, D. K. Georgiou, A. D. Joshi, and S. L. Hamilton, "Ryanodine receptors: structure, expression, molecular details, and function in calcium release," Cold Spring Harbor Perspectives in Biology, vol. 2, no. 11, article a003996, 2010.

[10] H. F. Deluca and G. W. Engstrom, "Calcium uptake by rat kidney mitochondria," Proceedings of the National Academy of Sciences of the United States of America, vol. 47, no. 11, pp. 1744-1750, 1961.

[11] F. D. Vasington and J. V. Murphy, " $\mathrm{Ca}^{++}$uptake by rat kidney mitochondria and its dependence on respiration and phosphorylation," Journal of Biological Chemistry, vol. 237, pp. 2670-2677, 1962.

[12] P. Mitchell, "Chemiosmotic coupling in oxidative and photosynthetic phosphorylation," Biological Reviews, vol. 41, no. 3, pp. 445-501, 1966.

[13] H. Rottenberg and A. Scarpa, "Calcium uptake and membrane potential in mitochondria," Biochemistry, vol. 13, no. 23, pp. 4811-4817, 1974.

[14] Y. Kirichok, G. Krapivinsky, and D. E. Clapham, "The mitochondrial calcium uniporter is a highly selective ion channel," Nature, vol. 427, no. 6972, pp. 360-364, 2004.

[15] C. Mammucari, A. Raffaello, D. Vecellio Reane, and R. Rizzuto, "Molecular structure and pathophysiological roles of the mitochondrial calcium uniporter," Biochimica et Biophysica Acta, vol. 1863, no. 10, pp. 2457-2464, 2016.

[16] R. Rizzuto, A. W. Simpson, M. Brini, and T. Pozzan, "Rapid changes of mitochondrial $\mathrm{Ca}^{2+}$ revealed by specifically targeted recombinant aequorin," Nature, vol. 358, no. 6384, pp. 325-327, 1992.

[17] R. Rizzuto, M. Brini, P. Pizzo, M. Murgia, and T. Pozzan, "Chimeric green fluorescent protein as a tool for visualizing subcellular organelles in living cells," Current Biology, vol. 5, no. 6, pp. 635-642, 1995.

[18] R. Rizzuto, P. Pinton, W. Carrington et al., "Close contacts with the endoplasmic reticulum as determinants of mitochondrial $\mathrm{Ca}^{2+}$ responses," Science, vol. 280, no. 5370, pp. 1763-1766, 1998.

[19] G. Csordás, A. P. Thomas, and G. Hajnóczky, "Quasisynaptic calcium signal transmission between endoplasmic reticulum and mitochondria," The EMBO Journal, vol. 18, no. 1, pp. 96-108, 1999.

[20] S. P. Mathupala and P. L. Pedersen, "Voltage dependent anion channel-1 (VDAC-1) as an anti-cancer target," Cancer Biology \& Therapy, vol. 9, no. 12, pp. 1053-1056, 2010.

[21] V.Shoshan-Barmatz, S. De, and A. Meir, "The mitochondrial voltage-dependent anion channel $1, \mathrm{Ca}^{2+}$ transport, apoptosis, and their regulation," Frontiers in Oncology, vol. 7, pp. 1-12, 2017.

[22] M. Madesh and G. Hajnoczky, "VDAC-dependent permeabilization of the outer mitochondrial membrane by superoxide induces rapid and massive cytochrome c release," The Journal of Cell Biology, vol. 155, no. 6, pp. 1003-1016, 2001.

[23] E. Rapizzi, P. Pinton, G. Szabadkai et al., "Recombinant expression of the voltage-dependent anion channel enhances the transfer of $\mathrm{Ca}^{2+}$ microdomains to mitochondria," The Journal of Cell Biology, vol. 159, no. 4, pp. 613-624, 2002.

[24] J. M. Baughman, F. Perocchi, H. S. Girgis et al., "Integrative genomics identifies MCU as an essential component of the mitochondrial calcium uniporter," Nature, vol. 476, no. 7360, pp. 341-345, 2011.

[25] D. De Stefani, A. Raffaello, E. Teardo, I. Szabò, and R. Rizzuto, "A forty-kilodalton protein of the inner membrane is the mitochondrial calcium uniporter," Nature, vol. 476, no. 7360, pp. 336-340, 2011.

[26] M. Patron, V. Checchetto, A. Raffaello et al., "MICU1 and MICU2 finely tune the mitochondrial $\mathrm{Ca}^{2+}$ uniporter by exerting opposite effects on MCU activity," Molecular Cell, vol. 53, no. 5, pp. 726-737, 2014.

[27] M. Plovanich, R. L. Bogorad, Y. Sancak et al., "MICU2, a paralog of MICU1, resides within the mitochondrial uniporter complex to regulate calcium handling," PLoS One, vol. 8, no. 2, article e55785, 2013.

[28] Y. Sancak, A. L. Markhard, T. Kitami et al., "EMRE is an essential component of the mitochondrial calcium uniporter complex," Science, vol. 342, no. 6164, pp. 1379-1382, 2013.

[29] K. Oxenoid, Y. Dong, C. Cao et al., "Architecture of the mitochondrial calcium uniporter," Nature, vol. 533, no. 7602, pp. 269-273, 2016.

[30] R. Baradaran, C. Wang, A. Francis Siliciano, S. Barstow long, R. Baradaran, and C. Wang, "Cryo-EM structures of fungal and metazoan mitochondrial calcium uniporters," Nature, vol. 559, no. 7715, pp. 580-584, 2018.

[31] J. Yoo, M. Wu, Y. Yin, M. A. Herzik, G. C. Lander, and S.-Y. Lee, "Cryo-EM structure of a mitochondrial calcium uniporter," Science, vol. 361, no. 6401, pp. 506-511, 2018.

[32] C. Fan, M. Fan, B. J. Orlando et al., "X-ray and cryo-EM structures of the mitochondrial calcium uniporter," Nature, vol. 559, no. 7715, pp. 575-579, 2018.

[33] N. X. X. Nguyen, J. P. P. Armache, C. Lee et al., "Cryo-EM structure of a fungal mitochondrial calcium uniporter," Nature, vol. 559, no. 7715, pp. 570-574, 2018.

[34] Y. Lee, C. K. Min, T. G. Kim et al., "Structure and function of the N-terminal domain of the human mitochondrial calcium uniporter," EMBO Reports, vol. 16, no. 10, pp. 1318-1333, 2015.

[35] E. Kovacs-Bogdan, Y. Sancak, K. J. Kamer et al., "Reconstitution of the mitochondrial calcium uniporter in yeast," Proceedings of the National Academy of Sciences, vol. 111, no. 24, pp. 8985-8990, 2014.

[36] H. Vais, K. Mallilankaraman, D. O. D. Mak et al., "EMRE is a matrix $\mathrm{Ca}^{2+}$ sensor that governs gatekeeping of the mitochondrial $\mathrm{Ca}^{2+}$ uniporter," Cell Reports, vol. 14, no. 3, pp. 403-410, 2016.

[37] T. Yamamoto, R. Yamagoshi, K. Harada et al., "Analysis of the structure and function of EMRE in a yeast expression system," Biochimica et Biophysica Acta (BBA) - Bioenergetics, vol. 1857, no. 6, pp. 831-839, 2016.

[38] A. Raffaello, D. De Stefani, D. Sabbadin et al., "The mitochondrial calcium uniporter is a multimer that can include a dominant-negative pore-forming subunit," The EMBO Journal, vol. 32, no. 17, pp. 2362-2376, 2013.

[39] K. J. Kamer and V. K. Mootha, "The molecular era of the mitochondrial calcium uniporter," Nature Reviews. Molecular Cell Biology, vol. 16, no. 9, pp. 545-553, 2015. 
[40] F. Perocchi, V. M. Gohil, H. S. Girgis et al., "MICU1 encodes a mitochondrial EF hand protein required for $\mathrm{Ca}^{+}$uptake," Nature, vol. 467, no. 7313, pp. 291-296, 2010.

[41] K. Mallilankaraman, P. Doonan, C. Cárdenas et al., "MICU1 is an essential gatekeeper for MCU-mediated mitochondrial $\mathrm{Ca}(2+)$ uptake that regulates cell survival," Cell, vol. 151, no. 3, pp. 630-644, 2012.

[42] C. V. Logan, G. Szabadkai, J. A. Sharpe et al., "Loss-offunction mutations in MICU1 cause a brain and muscle disorder linked to primary alterations in mitochondrial calcium signaling," Nature Genetics, vol. 46, no. 2, pp. 188-193, 2013.

[43] G. Csordás, T. Golenár, E. L. Seifert et al., "MICU1 controls both the threshold and cooperative activation of the mitochondrial $\mathrm{Ca}^{2+}$ uniporter," Cell Metabolism, vol. 17, no. 6, pp. 976-987, 2013.

[44] M. Paillard, G. Csordás, K.-T. Huang, P. Várnai, S. K. Joseph, and G. Hajnóczky, "MICU1 interacts with the D-ring of the MCU pore to control its $\mathrm{Ca}^{2+}$ flux and sensitivity to Ru360," Molecular Cell, vol. 72, no. 4, pp. 778-785.e3, 2018.

[45] M. Paillard, G. Csordás, G. Szanda et al., "Tissue-specific mitochondrial decoding of cytoplasmic $\mathrm{Ca}^{2+}$ signals is controlled by the stoichiometry of MICU1/2 and MCU," Cell Reports, vol. 18, no. 10, pp. 2291-2300, 2017.

[46] K. J. Kamer and V. K. Mootha, "MICU1 and MICU2 play nonredundant roles in the regulation of the mitochondrial calcium uniporter," EMBO Reports, vol. 15, no. 3, pp. 299307, 2014.

[47] C. Petrungaro, K. M. Zimmermann, V. Küttner et al., "The $\mathrm{Ca}^{2+}$-dependent release of the Mia40-induced MICU1-MICU2 dimer from MCU regulates mitochondrial $\mathrm{Ca}^{2+}$ uptake," Cell Metabolism, vol. 22, no. 4, pp. 721-733, 2015.

[48] D. Vecellio Reane, F. Vallese, V. Checchetto et al., "A MICU1 splice variant confers high sensitivity to the mitochondrial $\mathrm{Ca}^{2+}$ uptake machinery of skeletal muscle," Molecular Cell, vol. 64, no. 4, pp. 760-773, 2016.

[49] L. Wang, X. Yang, S. Li et al., "Structural and mechanistic insights into MICU1 regulation of mitochondrial calcium uptake," The EMBO Journal, vol. 33, no. 6, pp. 594-604, 2014.

[50] J. G. McCormack, A. P. Halestrap, and R. M. Denton, "Role of calcium ions in regulation of mammalian intramitochondrial metabolism," Physiological Reviews, vol. 70, no. 2, pp. 391425, 1990.

[51] M. Patron, V. Granatiero, and J. Espino, "MICU3 is a tissuespecific enhancer of mitochondrial calcium uptake," Cell Death \& Differentiation, vol. 26, no. 1, pp. 179-195, 2018.

[52] K. Mallilankaraman, C. Cárdenas, P. J. Doonan et al., "MCUR1 is an essential component of mitochondrial $\mathrm{Ca}^{2+}$ uptake that regulates cellular metabolism," Nature Cell Biology, vol. 14, no. 12, pp. 1336-1343, 2012.

[53] V. Paupe, J. Prudent, E. P. Dassa, O. Z. Rendon, and E. A. Shoubridge, "CCDC90A (MCUR1) is a cytochrome c oxidase assembly factor and not a regulator of the mitochondrial calcium uniporter," Cell Metabolism, vol. 21, no. 1, pp. 109-116, 2015.

[54] D. Chaudhuri, D. J. Artiga, S. A. Abiria, and D. E. Clapham, "Mitochondrial calcium uniporter regulator 1 (MCUR1) regulates the calcium threshold for the mitochondrial permeability transition," Proceedings of the National Academy of Sciences, vol. 113, no. 13, pp. E1872-E1880, 2016.
[55] F. Di Lisa and P. Bernardi, "A CaPful of mechanisms regulating the mitochondrial permeability transition," Journal of Molecular and Cellular Cardiology, vol. 46, no. 6, pp. 775780, 2009.

[56] L. Biasutto, M. Azzolini, I. Szabò, and M. Zoratti, “The mitochondrial permeability transition pore in AD 2016: an update," Biochim. Biophys. Acta - Mol. Cell Res., vol. 1863, no. 10, pp. 2515-2530, 2016.

[57] V. Giorgio, S. von Stockum, M. Antoniel et al., "Dimers of mitochondrial ATP synthase form the permeability transition pore," Proceedings of the National Academy of Sciences, vol. 110, no. 15, pp. 5887-5892, 2013.

[58] V. Giorgio, V. Burchell, M. Schiavone et al., " $\mathrm{Ca}{ }^{2+}$ binding to F-ATP synthase $\beta$ subunit triggers the mitochondrial permeability transition," EMBO Reports, vol. 18, no. 7, pp. 10651076, 2017.

[59] P. Bernardi, A. Rasola, M. Forte, and G. Lippe, "The mitochondrial permeability transition pore: channel formation by F-ATP synthase, integration in signal transduction, and role in pathophysiology," Physiological Reviews, vol. 95, no. 4, pp. 1111-1155, 2015.

[60] M. Bonora, C. Morganti, G. Morciano et al., "Mitochondrial permeability transition involves dissociation of $\mathrm{F}_{1} \mathrm{~F}_{\mathrm{O}}$ ATP synthase dimers and C-ring conformation," EMBO Reports, vol. 18, no. 7, pp. 1077-1089, 2017.

[61] J. He, H. C. Ford, J. Carroll, S. Ding, I. M. Fearnley, and J. E. Walker, "Persistence of the mitochondrial permeability transition in the absence of subunit c of human ATP synthase," Proceedings of the National Academy of Sciences, vol. 114, no. 13, pp. 3409-3414, 2017.

[62] J. He, J. Carroll, S. Ding, I. M. Fearnley, and J. E. Walker, "Permeability transition in human mitochondria persists in the absence of peripheral stalk subunits of ATP synthase," Proceedings of the National Academy of Sciences, vol. 114, no. 34, pp. 9086-9091, 2017.

[63] G. Morciano, C. Giorgi, M. Bonora et al., "Molecular identity of the mitochondrial permeability transition pore and its role in ischemia-reperfusion injury," Journal of Molecular and Cellular Cardiology, vol. 78, pp. 142-153, 2015.

[64] V. Giorgio, F. Fogolari, G. Lippe, and P. Bernardi, "OSCP subunit of mitochondrial ATP synthase: role in regulation of enzyme function and of its transition to a pore," British Journal of Pharmacology, 2018.

[65] M. Carraro, V. Giorgio, J. Sileikyte et al., "Channel formation by yeast F-ATP synthase and the role of dimerization in the mitochondrial permeability transition," The Journal of Biological Chemistry, vol. 289, no. 23, pp. 15980-15985, 2014.

[66] A. Nickel, M. Kohlhaas, and C. Maack, "Mitochondrial reactive oxygen species production and elimination," Journal of Molecular and Cellular Cardiology, vol. 73, pp. 2633, 2014.

[67] D. B. Zorov, M. Juhaszova, and S. J. Sollott, "Mitochondrial reactive oxygen species (ROS) and ROS-induced ROS release," Physiological Reviews, vol. 94, no. 3, pp. 909-950, 2014.

[68] L. A. Sena and N. S. Chandel, "Physiological roles of mitochondrial reactive oxygen species," Molecular Cell, vol. 48, no. 2, pp. 158-167, 2012.

[69] J. F. Turrens, "Mitochondrial formation of reactive oxygen species," The Journal of Physiology, vol. 552, no. 2, pp. 335344, 2003. 
[70] A. Görlach, K. Bertram, S. Hudecova, and O. Krizanova, "Calcium and ROS: a mutual interplay," Redox Biology, vol. 6, pp. 260-271, 2015.

[71] P. S. Brookes, Y. Yoon, J. L. Robotham, M. W. Anders, and S.-S. Sheu, "Calcium, ATP, and ROS: a mitochondrial love-hate triangle," American Journal of Physiology. Cell Physiology, vol. 287, no. 4, pp. C817-C833, 2004.

[72] Z. Dong, S. Shanmughapriya, D. Tomar et al., "Mitochondrial $\mathrm{Ca}^{2+}$ uniporter is a mitochondrial luminal redox sensor that augments MCU channel activity," Molecular Cell, vol. 65, no. 6, pp. 1014-1028.e7, 2017.

[73] Y. J. H. J. Taverne, A. J. J. C. Bogers, D. J. Duncker, and D. Merkus, "Reactive oxygen species and the cardiovascular system," Oxidative Medicine and Cellular Longevity, vol. 2013, Article ID 862423, 15 pages, 2013.

[74] S. Dey, A. Sidor, and B. O’Rourke, "Compartment-specific control of reactive oxygen species scavenging by antioxidant pathway enzymes," Journal of Biological Chemistry, vol. 291, no. 21, pp. 11185-11197, 2016.

[75] S. F. Steinberg, "Oxidative stress and sarcomeric proteins," Circulation Research, vol. 112, no. 2, pp. 393-405, 2013.

[76] J. R. Erickson, M. A. Joiner, X. Guan et al., "A dynamic pathway for calcium-independent activation of CaMKII by methionine oxidation," Cell, vol. 133, no. 3, pp. 462-474, 2008.

[77] A. Zima and L. Blatter, "Redox regulation of cardiac calcium channels and transporters," Cardiovascular Research, vol. 71, no. 2, pp. 310-321, 2006.

[78] M. Kohlhaas, A. G. G. Nickel, and C. Maack, "Mitochondrial energetics and calcium coupling in the heart," The Journal of Physiology, vol. 595, no. 12, pp. 3753-3763, 2017.

[79] S. Cortassa, M. A. A. Aon, B. O. Rourke et al., "A computational model integrating electrophysiology, contraction, and mitochondrial bioenergetics in the ventricular myocyte," Biophysical Journal, vol. 91, no. 4, pp. 1564-1589, 2006.

[80] R. S. Balaban, "Cardiac energy metabolism homeostasis: role of cytosolic calcium," Journal of Molecular and Cellular Cardiology, vol. 34, no. 10, pp. 1259-1271, 2002.

[81] I. Drago, D. De Stefani, R. Rizzuto, and T. Pozzan, "Mitochondrial $\mathrm{Ca}^{2+}$ uptake contributes to buffering cytoplasmic $\mathrm{Ca}^{2+}$ peaks in cardiomyocytes," Proceedings of the National Academy of Sciences of the United States of America, vol. 109, no. 32, pp. 12986-12991, 2012.

[82] M. Giacomello, I. Drago, M. Bortolozzi et al., " $\mathrm{Ca}^{2+}$ hot spots on the mitochondrial surface are generated by $\mathrm{Ca}^{2+}$ mobilization from stores, but not by activation of storeoperated $\mathrm{Ca}^{2+}$ channels," Molecular Cell, vol. 38, no. 2, pp. 280-290, 2010.

[83] F. Fieni, S. B. B. Lee, Y. N. N. Jan, and Y. Kirichok, "Activity of the mitochondrial calcium uniporter varies greatly between tissues," Nature Communications, vol. 3, no. 1, pp. 13171317, 2012.

[84] D. M. M. Bers, "Altered cardiac myocyte ca regulation in heart failure,” Physiology, vol. 21, no. 6, pp. 380-387, 2006.

[85] C. Maack, S. Cortassa, M. A. Aon, A. N. Ganesan, T. Liu, and O. Rourke, "Elevated cytosolic $\mathrm{Na}^{+}$decreases mitochondrial $\mathrm{Ca}^{2+}$ uptake during excitation-contraction coupling and impairs energetic adaptation in cardiac myocytes," Circulation Research, vol. 99, no. 2, pp. 172-182, 2009.

[86] S. M. Pogwizd, J. P. McKenzie, and M. E. Cain, "Mechanisms underlying spontaneous and induced ventricular arrhythmias in patients with idiopathic dilated cardiomyopathy," Circulation, vol. 98, no. 22, pp. 2404-2414, 1998.

[87] S. M. Pogwizd, M. Qi, W. Yuan, A. M. Samarel, and D. M. Bers, "Upregulation of $\mathrm{Na}^{+} / \mathrm{Ca}^{2+}$ exchanger expression and function in an arrhythmogenic rabbit model of heart failure," Circulation Research, vol. 85, no. 11, pp. 1009-1019, 1999.

[88] C. R. Weber, V. Piacentino, S. R. Houser, and D. M. Bers, "Dynamic regulation of sodium/calcium exchange function in human heart failure," Circulation, vol. 108, no. 18, pp. 2224-2229, 2003.

[89] A. A. Armoundas, I. A. Hobai, G. F. Tomaselli, R. L. Winslow, and B. O'Rourke, "Role of sodium-calcium exchanger in modulating the action potential of ventricular myocytes from normal and failing hearts," Circulation Research, vol. 93, no. 1, pp. 46-53, 2003.

[90] E. Bertero and C. Maack, "Calcium signaling and reactive oxygen species in mitochondria," Circulation Research, vol. 122, no. 10, pp. 1460-1478, 2018.

[91] R. H. Schwinger, J. Wang, K. Frank et al., "Reduced sodium pump $\alpha_{1}, \alpha_{3}$, and $\beta_{1}$-isoform protein levels and $\mathrm{Na}^{+}, \mathrm{K}^{+}$ATPase activity but unchanged $\mathrm{Na}^{+}-\mathrm{Ca}^{2+}$ exchanger protein levels in human heart failure," Circulation, vol. 99, no. 16, pp. 2105-2112, 1999.

[92] O. I. Shamraj, I. L. Grupp, G. Grupp et al., "Characterisation of $\mathrm{Na} / \mathrm{K}-\mathrm{ATPase}$, its isoforms, and the inotropic response to ouabain in isolated failing human hearts," Cardiovascular Research, vol. 27, no. 12, pp. 2229-2237, 1993.

[93] S. Despa, M. A. Islam, C. R. Weber, S. M. Pogwizd, and D. M. Bers, "Intracellular $\mathrm{Na}^{+}$concentration is elevated in heart failure but $\mathrm{Na} / \mathrm{K}$ pump function is unchanged," Circulation, vol. 105, no. 21, pp. 2543-2548, 2002.

[94] G. Michels, I. F. F. Khan, J. Endres-Becker et al., "Regulation of the human cardiac mitochondrial $\mathrm{Ca}^{2+}$ uptake by 2 different voltage-gated $\mathrm{Ca}^{2+}$ channels," Circulation, vol. 119, no. 18, pp. 2435-2443, 2009.

[95] M. Kohlhaas and C. Maack, "Adverse bioenergetic consequences of $\mathrm{Na}^{+}-\mathrm{Ca}^{2+}$ exchanger-mediated $\mathrm{Ca}^{2+}$ influx in cardiac myocytes," Circulation, vol. 122, no. 22, pp. $2273-$ 2280, 2010.

[96] M. Schieber and N. S. Chandel, "ROS function in redox signaling and oxidative stress," Current Biology, vol. 24, no. 10, pp. R453-R462, 2014.

[97] D. N. Granger and P. R. Kvietys, "Reperfusion injury and reactive oxygen species: the evolution of a concept," Redox Biology, vol. 6, pp. 524-551, 2015.

[98] G. C. Koenig, R. G. Rowe, S. M. Day et al., "MT1-MMPdependent remodeling of cardiac extracellular matrix structure and function following myocardial infarction," The American Journal of Pathology, vol. 180, no. 5, pp. 18631878, 2012.

[99] X. Pan, J. Liu, T. Nguyen et al., "The physiological role of mitochondrial calcium revealed by mice lacking the mitochondrial calcium uniporter," Nature Cell Biology, vol. 15, no. 12, pp. 1464-1472, 2013.

[100] K. M. Holmström, X. Pan, J. C. Liu et al., “Assessment of cardiac function in mice lacking the mitochondrial calcium uniporter," Journal of Molecular and Cellular Cardiology, vol. 85, pp. 178-182, 2016.

[101] J. Q. Kwong, X. Lu, R. N. Correll et al., "The mitochondrial calcium uniporter selectively matches metabolic output to 
acute contractile stress in the Heart," Cell Reports, vol. 12, no. 1, pp. 15-22, 2015.

[102] T. S. Luongo, J. P. Lambert, A. Yuan et al., "The mitochondrial calcium uniporter matches energetic supply with cardiac workload during stress and modulates permeability transition," Cell Reports, vol. 12, no. 1, pp. 23-34, 2015.

[103] Y. Wu, T. P. Rasmussen, O. M. Koval et al., "The mitochondrial uniporter controls fight or flight heart rate increases," Nature Communications, vol. 6, no. 1, p. 6081, 2015.

[104] T. P. Rasmussen, Y. Wu, M. A. Joiner et al., "Inhibition of MCU forces extramitochondrial adaptations governing physiological and pathological stress responses in heart," Proceedings of the National Academy of Sciences of the United States of America, vol. 112, no. 29, pp. 9129-9134, 2015.

[105] E. Murphy, X. Pan, T. Nguyen, J. Liu, K. M. Holmström, and T. Finkel, "Unresolved questions from the analysis of mice lacking MCU expression," Biochemical and Biophysical Research Communications, vol. 449, no. 4, pp. 384-385, 2014.

[106] T. Liu and B. O’Rourke, "Enhancing mitochondrial $\mathrm{Ca}^{2+}$ uptake in myocytes from failing hearts restores energy supply and demand matching," Circulation Research, vol. 103, no. 3, pp. 279-288, 2008.

[107] Y. Song, J. C. Shryock, S. Wagner, L. S. Maier, and L. Belardinelli, "Blocking late sodium current reduces hydrogen peroxide-induced arrhythmogenic activity and contractile dysfunction," The Journal of Pharmacology and Experimental Therapeutics, vol. 318, no. 1, pp. 214-222, 2006.

[108] O. Zeitz, A. E. Maass, P. Van Nguyen et al., "Hydroxyl radical-induced acute diastolic dysfunction is due to calcium overload via reverse-mode $\mathrm{Na}(+)-\mathrm{Ca}(2+)$ exchange," Circulation Research, vol. 90, no. 9, pp. 988-995, 2002.

[109] M.-L. A. Joiner, O. M. Koval, J. Li et al., "CaMKII determines mitochondrial stress responses in heart," Nature, vol. 491, no. 7423, pp. 269-273, 2012.

[110] F. Fieni, D. E. Johnson, A. Hudmon, and Y. Kirichok, "Mitochondrial $\mathrm{Ca}^{2+}$ uniporter and CaMKII in heart," Nature, vol. 513, no. 7519, pp. E1-E2, 2014.

[111] E. Barbieri and P. Sestili, "Reactive oxygen species in skeletal muscle signaling," Journal of Signal Transduction, vol. 2012, Article ID 982794, 17 pages, 2012.

[112] M. L. Urso and P. M. Clarkson, "Oxidative stress, exercise, and antioxidant supplementation," Toxicology, vol. 189, no. 1-2, pp. 41-54, 2003.

[113] S. Cortassa, B. O’Rourke, and M. A. Aon, "Redox-optimized ROS balance and the relationship between mitochondrial respiration and ROS," Biochimica et Biophysica Acta (BBA) Bioenergetics, vol. 1837, no. 2, pp. 287-295, 2014.

[114] M. A. Aon, S. Cortassa, and B. O'Rourke, "Redox-optimized ROS balance: a unifying hypothesis," Biochimica et Biophysica Acta (BBA) - Bioenergetics, vol. 1797, no. 6-7, pp. 865877, 2010.

[115] S. Messina, D. Altavilla, M. Aguennouz et al., "Lipid peroxidation inhibition blunts nuclear factor- $\kappa \mathrm{B}$ activation, reduces skeletal muscle degeneration, and enhances muscle function in $m d x$ mice," The American Journal of Pathology, vol. 168, no. 3, pp. 918-926, 2006.

[116] A. Toscano, S. Messina, G. M. Campo et al., "Oxidative stress in myotonic dystrophy type 1," Free Radical Research, vol. 39, no. 7, pp. 771-776, 2005.
[117] S. Fulle, F. Protasi, G. Di Tano et al., "The contribution of reactive oxygen species to sarcopenia and muscle ageing," Experimental Gerontology, vol. 39, no. 1, pp. 17-24, 2004.

[118] M. Buck and M. Chojkier, "Muscle wasting and dedifferentiation induced by oxidative stress in a murine model of cachexia is prevented by inhibitors of nitric oxide synthesis and antioxidants," The EMBO Journal, vol. 15, no. 8, pp. 1753-1765, 1996.

[119] J. S. Moylan and M. B. Reid, "Oxidative stress, chronic disease, and muscle wasting," Muscle \& Nerve, vol. 35, no. 4, pp. 411-429, 2007.

[120] R. Robinson, D. Carpenter, M.-A. Shaw, J. Halsall, and P. Hopkins, "Mutations in RYR1 in malignant hyperthermia and central core disease," Human Mutation, vol. 27, no. 10, pp. 977-989, 2006.

[121] D. Schneiderbanger, S. Johannsen, N. Roewer, and F. Schuster, "Management of malignant hyperthermia: diagnosis and treatment," Therapeutics and Clinical Risk Management, vol. 10, pp. 355-362, 2014.

[122] P. Szentesi, C. Collet, S. Sárközi et al., "Effects of dantrolene on steps of excitation-contraction coupling in mammalian skeletal muscle fibers," The Journal of General Physiology, vol. 118, no. 4, pp. 355-376, 2001.

[123] A. Michelucci, A. De Marco, F. A. Guarnier, F. Protasi, and S. Boncompagni, "Antioxidant treatment reduces formation of structural cores and improves muscle function in RYR1 ${ }^{\mathrm{Y} 522 \mathrm{~S} / \mathrm{WT}}$ mice," Oxidative Medicine and Cellular Longevity, vol. 2017, Article ID 6792694, 15 pages, 2017.

[124] W. J. Durham, P. Aracena-Parks, C. Long et al., "RyR1 S-nitrosylation underlies environmental heat stroke and sudden death in Y522S RyR1 knockin mice," Cell, vol. 133, no. 1, pp. 53-65, 2008.

[125] M. Kelly-Worden and E. Thomas, "Mitochondrial dysfunction in Duchenne muscular dystrophy," Open Journal of Endocrine and Metabolic Diseases, vol. 4, no. 8, pp. 211218, 2014.

[126] J. C. Liu, J. Liu, K. M. Holmström et al., "MICU1 serves as a molecular gatekeeper to prevent in vivo mitochondrial calcium overload," Cell Reports, vol. 16, no. 6, pp. 1561-1573, 2016.

[127] T. Calì, D. Ottolini, and M. Brini, "Mitochondrial $\mathrm{Ca}^{2+}$ and neurodegeneration," Cell Calcium, vol. 52, no. 1, pp. 73-85, 2012.

[128] A. Kumar, K. Bodhinathan, and T. C. Foster, "Susceptibility to calcium dysregulation during brain aging," Frontiers in Aging Neuroscience, vol. 1, p. 2, 2009.

[129] M. Brini, T. Calì, D. Ottolini, and E. Carafoli, "Neuronal calcium signaling: function and dysfunction," Cellular and Molecular Life Sciences, vol. 71, no. 15, pp. 2787-2814, 2014.

[130] M. J. Berridge, "Neuronal calcium signaling," Neuron, vol. 21, no. 1, pp. 13-26, 1998.

[131] I. Llorente-Folch, C. B. Rueda, B. Pardo, G. Szabadkai, M. R. Duchen, and J. Satrustegui, "The regulation of neuronal mitochondrial metabolism by calcium," The Journal of Physiology, vol. 593, no. 16, pp. 3447-3462, 2015.

[132] T. F. Beckhauser, J. Francis-Oliveira, and R. De Pasquale, "Reactive oxygen species: physiological and physiopathological effects on synaptic plasticity," Journal of Experimental Neuroscience, vol. 10s1, 2016. 
[133] J. Chao, L. Yang, S. Buch, and L. Gao, "Angiotensin II increased neuronal stem cell proliferation: role of AT2R," PLoS One, vol. 8, no. 5, article e63488, 2013.

[134] E. Topchiy, E. Panzhinskiy, W. S. T. Griffin, S. W. Barger, M. Das, and W. M. Zawada, "Nox4-generated superoxide drives angiotensin II-induced neural stem cell proliferation," Developmental Neuroscience, vol. 35, no. 4, pp. 293-305, 2013.

[135] C. Hidalgo and A. Arias-Cavieres, "Calcium, reactive oxygen species, and synaptic plasticity," Physiology, vol. 31, no. 3, pp. 201-215, 2016.

[136] C. A. Massaad and E. Klann, "Reactive oxygen species in the regulation of synaptic plasticity and memory," Antioxidants \& Redox Signaling, vol. 14, no. 10, pp. 2013-2054, 2011.

[137] Z. Li, G. Ji, and V. Neugebauer, "Mitochondrial reactive oxygen species are activated by mGluR5 through IP3 and activate ERK and PKA to increase excitability of amygdala neurons and pain behavior," The Journal of Neuroscience, vol. 31, no. 3, pp. 1114-1127, 2011.

[138] R. De Pasquale, T. F. Beckhauser, M. S. Hernandes, and L. R. Giorgetti Britto, "LTP and LTD in the visual cortex require the activation of NOX2," The Journal of Neuroscience, vol. 34, no. 38, pp. 12778-12787, 2014.

[139] H. Fujii and T. Hirano, "Calcineurin regulates induction of late phase of cerebellar long-term depression in rat cultured Purkinje neurons," The European Journal of Neuroscience, vol. 16, no. 9, pp. 1777-1788, 2002.

[140] H. Y. Kim, K. Y. Lee, Y. Lu et al., "Mitochondrial $\mathrm{Ca}^{2+}$ uptake is essential for synaptic plasticity in pain," The Journal of Neuroscience, vol. 31, no. 36, pp. 12982-12991, 2011.

[141] J. A. Dykens, "Isolated cerebral and cerebellar mitochondria produce free radicals when exposed to elevated $\mathrm{Ca}^{2+}$ and $\mathrm{Na}^{+}$: implications for neurodegeneration," Journal of Neurochemistry, vol. 63, no. 2, pp. 584-591, 1994.

[142] I. J. Reynolds and T. G. Hastings, "Glutamate induces the production of reactive oxygen species in cultured forebrain neurons following NMDA receptor activation," The Journal of Neuroscience, vol. 15, no. 5, pp. 3318-3327, 1995.

[143] H. She, Q. Yang, K. Shepherd et al., "Direct regulation of complex I by mitochondrial MEF2D is disrupted in a mouse model of Parkinson disease and in human patients," The Journal of Clinical Investigation, vol. 121, no. 3, pp. 930940, 2011.

[144] G. H. Kim, J. E. Kim, S. J. Rhie, and S. Yoon, "The role of oxidative stress in neurodegenerative diseases," Experimental Neurobiology, vol. 24, no. 4, pp. 325-340, 2015.

[145] J. Qiu, Y.-W. Tan, A. M. Hagenston et al., "Mitochondrial calcium uniporter Mcu controls excitotoxicity and is transcriptionally repressed by neuroprotective nuclear calcium signals," Nature Communications, vol. 4, no. 1, p. 2034, 2013.

[146] N. N. Pavlova and C. B. Thompson, "The emerging hallmarks of cancer metabolism," Cell Metabolism, vol. 23, no. 1, pp. 27-47, 2016.

[147] S. Marchi and P. Pinton, "Alterations of calcium homeostasis in cancer cells," Current Opinion in Pharmacology, vol. 29, pp. 1-6, 2016.

[148] S. Marchi, L. Lupini, S. Patergnani et al., "Downregulation of the mitochondrial calcium uniporter by cancer-related miR-25," Current Biology, vol. 23, no. 1, pp. 58-63, 2013.

[149] A. Tosatto, R. Sommaggio, C. Kummerow et al., "The mitochondrial calcium uniporter regulates breast cancer progression via HIF-1 $\alpha$, EMBO Molecular Medicine, vol. 8, no. 5, pp. 569-585, 2016.

[150] G.-Y. Liou and P. Storz, "Reactive oxygen species in cancer," Free Radical Research, vol. 44, no. 5, pp. 479-496, 2010.

[151] S. Marchi, M. Corricelli, A. Branchini et al., “Akt-mediated phosphorylation of MICU1 regulates mitochondrial $\mathrm{Ca}^{2+}$ levels and tumor growth," The EMBO Journal, vol. 38, no. 2, article e99435, 2019.

[152] A. Gastaldello, H. Callaghan, P. Gami, and M. Campanella, " $\mathrm{Ca}^{2+}$-dependent autophagy is enhanced by the pharmacological agent PK11195," Autophagy, vol. 6, no. 5, pp. 607$613,2010$.

[153] J. M. Vicencio, C. Ortiz, A. Criollo et al., "The inositol 1,4,5-trisphosphate receptor regulates autophagy through its interaction with Beclin 1," Cell Death and Differentiation, vol. 16, no. 7, pp. 1006-1017, 2009.

[154] A. Rimessi, M. Bonora, S. Marchi et al., "Perturbed mitochondrial $\mathrm{Ca}^{2+}$ signals as causes or consequences of mitophagy induction," Autophagy, vol. 9, no. 11, pp. 1677-1686, 2013.

[155] J. P. Bernardini, M. Lazarou, and G. Dewson, "Parkin and mitophagy in cancer," Oncogene, vol. 36, no. 10, pp. 13151327, 2017.

[156] M. C. Picchio, E. S. Martin, R. Cesari et al., "Alterations of the tumor suppressor gene Parkin in non-small cell lung cancer," Clinical Cancer Research, vol. 10, no. 8, pp. 2720-2724, 2004.

[157] F.-M. Kong, M. S. Anscher, M. K. Washington, J. K. Killian, and R. L. Jirtle, "M6P/IGF2R is mutated in squamous cell carcinoma of the lung," Oncogene, vol. 19, no. 12, pp. 15721578, 2000.

[158] S. R. Denison, F. Wang, N. A. Becker et al., "Alterations in the common fragile site gene Parkin in ovarian and other cancers," Oncogene, vol. 22, no. 51, pp. 8370-8378, 2003.

[159] S. Saito, S. Sirahama, M. Matsushima et al., "Definition of a commonly deleted region in ovarian cancers to a $300-\mathrm{kb}$ segment of chromosome 6q27," vol. 56, no. 24, pp. 5586-5589, 1996.

[160] H. Alder, M. Shimizu, A. Drusco et al., "Parkin, a gene implicated in autosomal recessive juvenile parkinsonism, is a candidate tumor suppressor gene on chromosome 6q25-q27," Proceedings of the National Academy of Sciences, vol. 100, no. 10, pp. 5956-5961, 2003.

[161] C. Giorgi, C. Agnoletto, A. Bononi et al., "Mitochondrial calcium homeostasis as potential target for mitochondrial medicine," Mitochondrion, vol. 12, no. 1, pp. 77-85, 2012. 


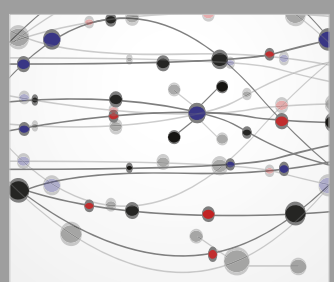

The Scientific World Journal
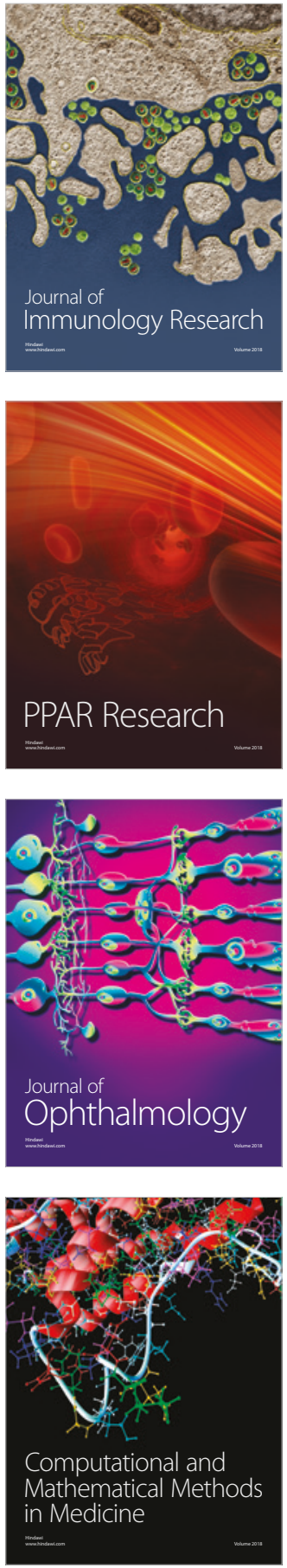

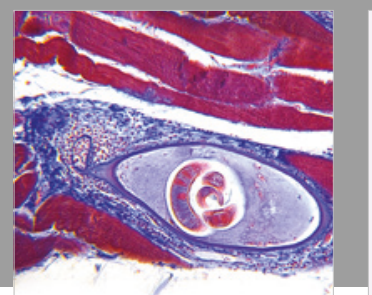

Gastroenterology Research and Practice

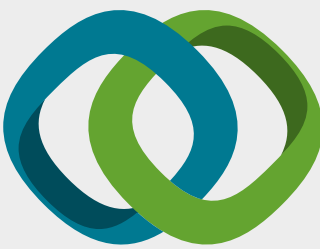

\section{Hindawi}

Submit your manuscripts at

www.hindawi.com
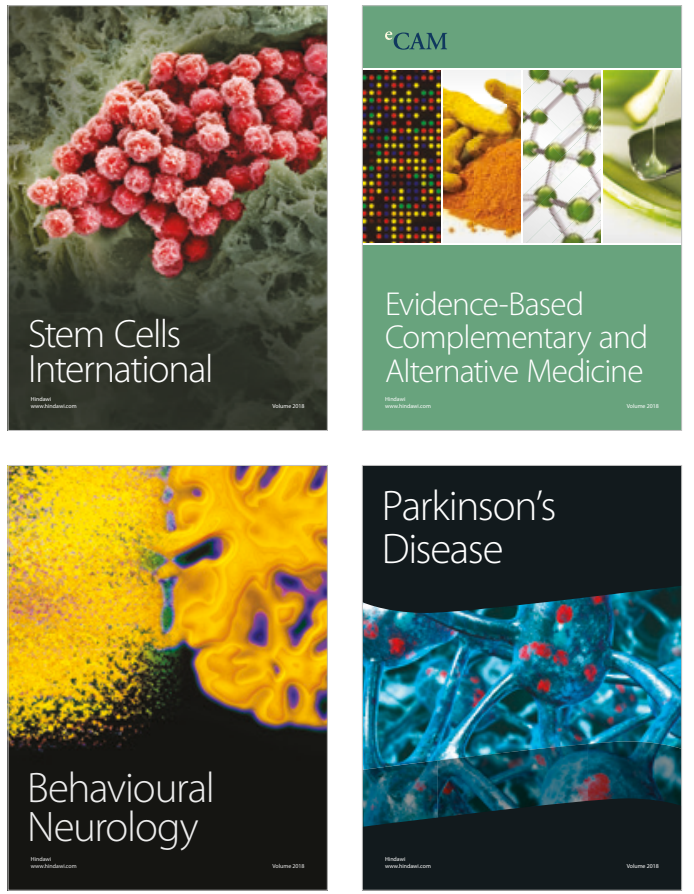

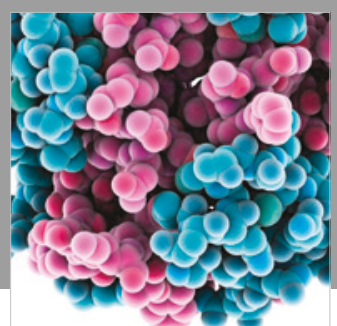

ournal of

Diabetes Research

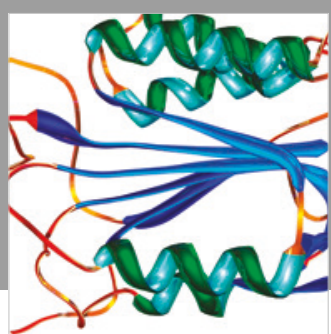

Disease Markers
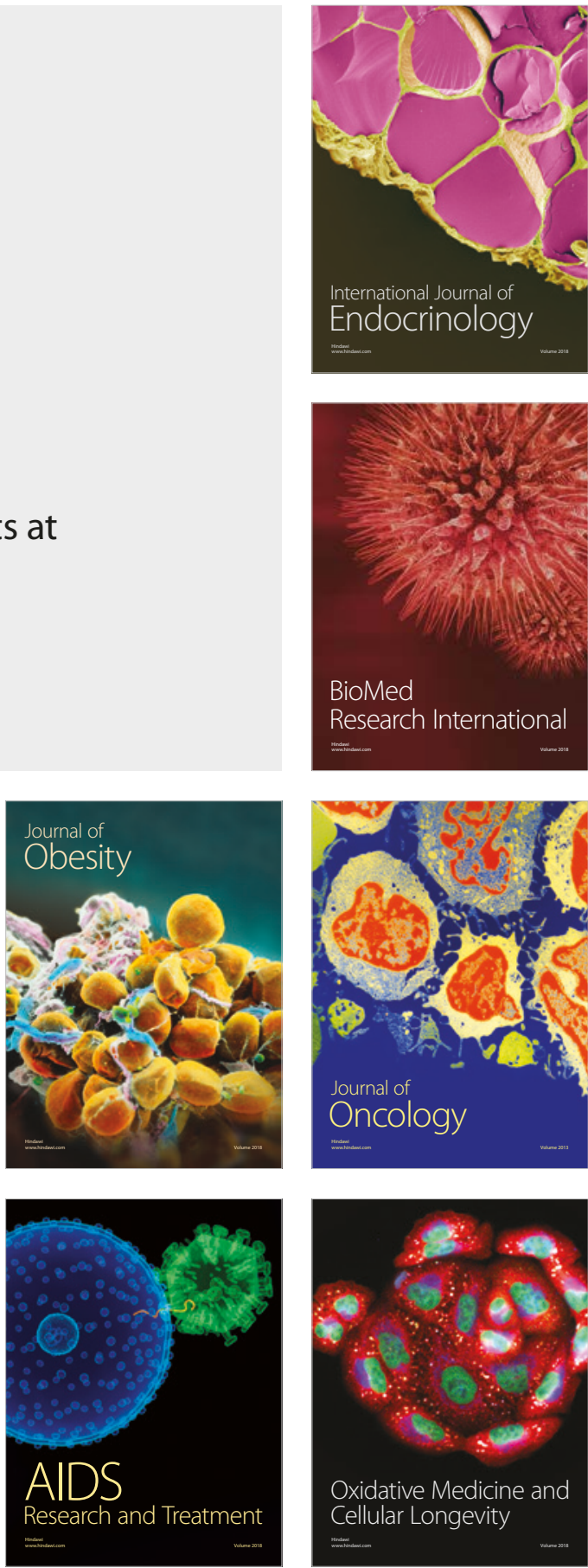